\title{
Low Temperature Phase Diagrams for Quantum Perturbations of Classical Spin Systems
}

\author{
C. Borgs ${ }^{1, \star}$, R. Kotecký ${ }^{2, \star \star}$, D. Ueltschi ${ }^{3}$ \\ 1 School of Mathematics, Institute for Advanced Study, Princeton, NJ 08540, USA \\ 2 Centre de Physique Théorique, CNRS, Marseille, France \\ ${ }^{3}$ Institut de Physique Théorique, EPF Lausanne, Switzerland. E-mail: ueltschi@elda.epfl ch
}

Received: 10 June 1995/Accepted: 14 February 1996

Abstract: We consider a quantum spin system with Hamiltonian

$$
H=H^{(0)}+\lambda V
$$

where $H^{(0)}$ is diagonal in a basis $|s\rangle=\bigotimes_{x}\left|s_{x}\right\rangle$ which may be labeled by the configurations $s=\left\{s_{x}\right\}$ of a suitable classical spin system on $\mathbb{Z}^{d}$,

$$
H^{(0)}|s\rangle=H^{(0)}(s)|s\rangle
$$

We assume that $H^{(0)}(s)$ is a finite range Hamiltonian with finitely many ground states and a suitable Peierls condition for excitations, while $V$ is a finite range or exponentially decaying quantum perturbation. Mapping the $d$ dimensional quantum system onto a classical contour system on a $d+1$ dimensional lattice, we use standard Pirogov-Sinai theory to show that the low temperature phase diagram of the quantum spin system is a small perturbation of the zero temperature phase diagram of the classical Hamiltonian $H^{(0)}$, provided $\lambda$ is sufficiently small. Our method can be applied to bosonic systems without substantial change. The extension to fermionic systems will be discussed in a subsequent paper.

\section{Introduction}

1.1. General ideas. Many models of classical statistical mechanics provide examples of first-order phase transitions and phase coexistence at low temperatures. It became clear already from the first proof of such a transition for the Ising model by the Peierls argument [Pei36, Gri64, Dob65] that a convenient tool for the study of phase coexistence and first-order phase transitions is a representation in terms of configurations of geometrical objects - contours. This has been systematically

\footnotetext{
${ }^{\star}$ Heisenberg Fellow, on leave from Institut für Theoretische Physik, Freie Universität Berlin E-mail: borgs@dirac physik.fu-berlin de

$\star \star$ On leave of absence from Center for Theoretical Study, Charles University, Praha Partly supported by the grants GAČR 202/93/0449 and GAUK 376 E-mail: kotecky@cucc ruk cunicz
} 
developed in Pirogov-Sinai theory [PS75, Sin82], see also [KP84, Zah84, BI89], which allows one to prove these phenomena for a wide class of models, with or without symmetry assumptions on the coexisting phases.

For quantum spin systems, the theory of first-order phase transitions and phase coexistence is much less developed. While several papers deal with this problem in the presence of a symmetry relating the two phases, [Gin69, Ken85], no general theory is known which provides a systematic approach to quantum spin systems once the symmetry constraint is relaxed

In this paper we propose to develop such a theory for low temperature quantum spin systems which are small perturbations of suitable classical systems To be more precise, we assume that the Hamiltonian of the system is of the form

$$
H=H^{(0)}+i V,
$$

where $H^{(0)}$ is diagonal in a basis $|s\rangle=\bigotimes_{\gamma}\left|s_{\gamma}\right\rangle$ which may be labelled by the configurations $s=\left\{s_{r}\right\}$ of a classical spin system with finite single spin space $S=\{1, \quad .,|S|\}$ and Hamiltonian

$$
\left\langle s\left|H^{(0)}\right| s\right\rangle=H^{(0)}(s),
$$

while $V$ is a local or exponentially decaying quantum perturbation,

$$
V=\sum_{A} V_{A}
$$

where the sum goes over connected sets $A$ and $V_{A}$ is an arbitrary operator on $\mathscr{H}_{A}=$ $\bigotimes_{x \in A} \mathscr{H}_{\lambda}$, except for the constraint that its norm $\left\|V_{A}\right\|$ is exponentially decaying with the size of $A$.

Assuming that the classical system has a contour representation in $d$ dimensions that allows to apply the methods of Pirogov-Sinai theory for sufficiently low temperatures, we propose to study the quantum perturbation of this system by mapping it into a suitable contour system in $d+1$ dimensions, which can again be analysed by the methods of Pirogov-Sinai theory. Actually, our method is very similar to the method developed in [Bor88], were this strategy was used to develop weak coupling cluster expansions for lattice gauge theories with discrete gauge group and continuous time.

Our approach differs, however, from that used by Ginibre [Gin69] and Kennedy [Ken85] In order to explain the main difference, let us first recall their method. It is based on the idea of developing the density matrix $e^{-\beta H}$ of the model (1.1) arround the unperturbed matrix $e^{-\beta H^{(0)}}$ using Trotter's formula

$$
e^{-\beta H}=\lim _{n \rightarrow \infty}\left(e^{-(\beta / n) H^{(0)}}\left(1-\frac{\beta}{n} V\right)\right)^{n} .
$$

While the leading term of this expansion gives the partition function of the classical spin system at temperature $\beta$, the expansion of $V$ according to (1.3) will introduce transitions between classical contours at various times, leading therefore to a representation in terms of "quantum contours" on $\mathbb{Z}^{d} \times[0, \beta]$.

In the symmetric case considered in [Ken85] and [Gin69], these quantum contours could be controlled with affordable effort using standard methods. The 
asymmetric situation considered here, however, obviously requires significant modifications involving something like a "Quantum Pirogov-Sinai" theory. Such an approach is currently being pursued by Datta, Fernandez and Fröhlich [DFF95], leading to results very similar to those presented in this paper ${ }^{1}$.

Here, we follow an alternative approach, motivated by [Bor88]. The main idea is not to consider contours on $\mathbb{Z}^{d} \times[0, \beta]$, but to use a suitable blocked approach, which allows to map a $d$-dimensional quantum system onto a classical contour system on the $(d+1)$-dimensional block lattice. As a consequence, our results make it possible to apply directly the usual Pirogov-Sinai theory to quantum spin systems as well, thereby allowing to analyze questions concerning the low temperature phase structure, finite size scaling, analyticity properties, etc. using the well developed machinery of standard Pirogov-Sinai theory.

1.2 Contour representations of quantum lattice models. In the remaining part of this introduction, we present the main ideas of our approach. In the first step, we rewrite the partition function $Z=\operatorname{Tr} e^{-\beta H}$ of the quantum spin system as

$$
Z=\operatorname{Tr} T^{M} \text { where } T=e^{-\tilde{\beta} H} \text { and } \beta=M \tilde{\beta}
$$

with an integer $M$ to be chosen later. We then expand the partition function $Z$ of the quantum system around the partition function $Z^{\text {class }}=\operatorname{Tr} e^{-\beta H^{(0)}}$ of the classical spin system using the Duhamel formula (a reference concerning the Duhamel formula is e.g. [SS76]) for the transfer matrix $T=e^{-\tilde{\beta} H}$. Introducing the family $\mathscr{A}_{0}$ of all sets $A$ contributing to (1.3), the Duhamel expansion gives

$$
T=e^{-\tilde{\beta} H^{(0)}-\tilde{\beta} \lambda \sum_{A \in \mathscr{S}_{0}} V_{A}}=\sum_{\mathbf{n}}\left[\prod_{A \in \mathscr{A}_{0}} \frac{(-\lambda)^{n_{A}}}{n_{A} !} \int_{0}^{\tilde{\beta}} d \tau_{A}^{1} \cdots d \tau_{A}^{n_{A}}\right] T(\tau, \mathbf{n}),
$$

where $\mathbf{n}$ is an multiindex, $\mathbf{n}: \mathscr{A}_{0} \rightarrow\{0,1,2, \ldots\}$, and $T(\tau, \mathbf{n})$ is obtained from $T^{(0)}=$ $e^{-\tilde{\beta} H}$ by "inserting" the operator $V_{A}$ at the times $\tau_{A}^{1}, \ldots, \tau_{A}^{n_{A}}$, see Sect. 2 for the precise definition. Next, we resum (1.6) to obtain the expansion

$$
T=\sum_{B} T(B)
$$

where

$$
T(B)=\sum_{\substack{\mathscr{A}=\left\{A_{1},, A_{k}\right\} \\ \cup_{l} A_{l}=B}} \tilde{T}(\mathscr{A}),
$$

with

$$
\tilde{T}(\mathscr{A})=\sum_{\mathbf{n}: \operatorname{supp} \mathbf{n}=\mathscr{A}}\left[\prod_{A \in \mathscr{A}_{0}} \frac{(-\lambda)^{n_{A}}}{n_{A} !} \int_{0}^{\tilde{\beta}} d \tau_{A}^{1} \cdots d \tau_{A}^{n_{A}}\right] T(\tau, \mathbf{n}) .
$$

\footnotetext{
${ }^{1}$ Results of this type were announced some time ago in [Pir78] However, a detailed discussion and proofs have never been presented
} 
Using the basis $|s\rangle$ to rewrite (1.5) as

$$
Z=\sum_{s^{(1)}, s^{(M)}}\left\langle s^{(1)}|T| s^{(2)}\right\rangle \cdot\left\langle s^{(M)}|T| s^{(1)}\right\rangle,
$$

and inserting the formula (1.7) to expand $T$ around $T^{(0)}$, we obtain

$$
Z=\sum_{\substack{s^{(1)}, s^{(M)} \\ B^{(1)}, \quad, B^{(M)}}} \prod_{t=1}^{M}\left\langle s^{(t-1)}\left|\tilde{T}\left(B^{(t)}\right)\right| s^{(t)}\right\rangle,
$$

where we have identified $s^{(0)}$ and $s^{(M)}$

At this point, the quantum spin system is easily mapped to a classical contour system in $d+1$ dimensions. Before doing so, let us discuss the expansion (19) from a more heuristic point of view. Starting with the leading term in $B^{(1)}, \ldots, B^{(M)}$, namely the term where all $B^{(t)}$ are empty, the matrices $T\left(B^{(t)}\right)$ reduce to the unperturbed transfer matrix $T^{(0)}$, which implies that only the term with $s^{(1)} \equiv s^{(2)} \equiv \cdots \equiv s^{(M)}$ contributes to the sum over $s^{(1)}, \ldots, s^{(M)}$, giving the partition function of the classical spin system at the inverse temperature $\beta$. A non-empty set $B^{(t)}$, on the other hand, corresponds to the insertion of one or several operators $V_{A}, A \subset B^{(t)}$, inducing transitions between different classical states $s^{(t-1)}$ and $s^{(t)}$ It should be noted, however, that for a fixed set of $B^{(t)}$ 's only those spin configurations $s^{(1)} \equiv s^{(2)} \equiv \cdots \equiv s^{(M)}$ contribute to (1.9), for which $s^{(t-1)}$ and $s^{(t)}$ are identical on all points $x$ for which $x \notin B^{(t)}$.

In order to rewrite (19) in terms of contours, we assign contours to "configurations" specified by $s^{(1)}, ., s^{(M)}$ and $B^{(1)}, \quad, B^{(M)}$. Namely, we introduce elementary cubes as the unit closed cubes $C \subset \mathbb{R}^{d+1}$ with centers $(x, t)$, where $t \in\{1,2, \ldots, M\}$ and $x \in \mathbb{Z}^{d}$. We say that an elementary cube $C$ with center $(x, t), x \in \mathbb{Z}^{d}$, lies in the $t^{\text {th }}$ time slice, we say that it is in a quantum excited state if $x \in B^{(t)}$, while we say it is in a classical state if this is not the case Consider now a cube $C$ in the $t^{\text {th }}$ time slice which is in a classical state Then $s^{(t-1)}$ and $s^{(t)}$ must assume the same value $s_{x} \in S$ on the corresponding point $x \in \mathbb{Z}^{d}$, and we say that the cube $C$ is in the (classical) state $s_{r}$

In order to explain our definition of contours, let us assume for the purpose of this introduction that $H^{(0)}(s)$ is the Hamiltonian of a classical spin system with nearest neighbor interactions, and that the contours of the classical system correspond to bonds $\langle x y\rangle$ for which $s_{x} \neq s_{y}$ (see Sect. 2 for the more general case) We then say that a cube $C$ is part of the ground state region $W_{m}, m \in S$, if it is in the classical state $m$, and if all neighboring cubes in the same time slice are in the classical state $m$ as well All cubes which are not part of a ground state region are called excited As usual, we define a contour as a connected component of the set of excited cubes.

Resumming all terms in (1.9) which lead to the same set of ground state regions and contours, we finally obtain $Z$ as a sum over sets $\left\{Y_{1}, \ldots, Y_{n}\right\}$ of non-overlapping contours Given our definition of excited cubes, it is an easy exercise to show that the weight of each such configuration factors into a product of contour activities $\rho\left(Y_{l}\right)$ and ground state terms $e^{-\tilde{\beta} e_{m}\left|W_{m}\right|}$, where $e_{m}$ is the classical ground state energy of the ground state $m$, while $\left|W_{m}\right|$ is the number of cubes which are in the ground state $m$ This gives the representation

$$
Z=\sum_{\left\{Y_{1}, Y_{n}\right\}} \prod_{i} \rho\left(Y_{l}\right) \prod_{m} e^{-\tilde{\beta} e_{m}\left|W_{m}\right|}
$$


which is exactly of the same form as the contour representation of a classical spin system. We can therefore apply standard Pirogov-Sinai theory to analyze the quantum spin system considered here, provided we can prove a bound of the form

$$
|\rho(Y)| \leqq e^{-\gamma|Y|} e^{-\tilde{\beta} e_{0}|Y|},
$$

where $\gamma$ is a sufficiently large constant, and $e_{0}=\min _{m} e_{m}$.

Observing that cubes in a quantum excitation are supressed by a small factor proportional to $\lambda \tilde{\beta}$, while excited classical cubes are exponentially suppressed by a "classical" contour energy proportional to $\tilde{\beta}$, such a bound can easily be proven, see Sect. 4 for the details.

Notice that the weights $\rho(Y)$ are in general complex. The version of the PirogovSinai theory to be used thus must deal with this fact. Actually, such a case has been discussed in [BI89] and we are closely following their approach.

A novel feature of the models considered here stems from the fact that the resulting classical model resides in a finite slab of thickness proportional to $\beta$; actually we should talk of a cylinder because of the periodic boundary conditions. It is therefore not possible to directly apply standard Pirogov-Sinai theory. Actually, this gives rise to an interesting problem in both quantum and classical spin systems: dimensional crossover for first order phase transitions. We will not discuss the physics of dimensional crossover in this paper, but the technical modifications needed to deal with finite temperature quantum spin systems do actually provide the necessary framework to deal with this problem as well.

The organization of this paper is as follows: In the next section, we state our main assumptions and results. In Sect. 3, we derive the contour representation (1.10), proving in particular the needed factorization of contour activities. In Sect. 4 we prove the exponential decay of the contour activities. Section 5 is devoted to the discussion of the resulting contour model including the discussion of modifications to Pirogov-Sinai theory on a finite slab. In Sect. 6 we discuss expectation values of local observables, and in Sect. 7 we combine the results of the preceding section to prove the theorems stated in Sect. 2. Details of the necessary modifications to Pirogov-Sinai theory on a finite slab are deferred to an appendix.

We close this introduction with a discussion of possible extensions. We recall that we assumed that $H^{(0)}$ is diagonal in a basis $|s\rangle=\bigotimes_{x}\left|s_{x}\right\rangle$, where $s_{x}$ lies in a finite spin space $S$. While this is a natural setting for quantum spin systems as, e.g. the anisotropic quantum Heisenberg model, it is not for the discussion of bosonic or fermionic lattice gases. In this situation, a typical choice for $H^{(0)}$ would be an operator which is diagonal in the usual bosonic or fermionic Fock representation where basis vectors are characterized by eigenvalues of the corresponding number operators $n_{x}$. While the corresponding classical system still has a finite state space $\left(n_{x}=n_{\uparrow}+n_{\downarrow}=0,1,2\right)$ in the fermionic case, bosons now give rise to a state space which contains infinitely many classical states per site. In most applications, however, this is not a serious problem, because the hamiltonian $H^{(0)}$ suppresses high values of $n_{x}$ (or, in the usual field representation, high values of the boson field $\phi_{x}$ ). Our methods and results are therefore applicable to bosonic lattice gases without major modifications.

For fermions, on the other hand, the antisymmetrization of the wave function leads to sign problems in the contour representation (1.10) which have to be dealt with carefully. While this is a priori not obvious at all, it turns out, however, that 
fermion signs do not spoil the factorization properties needed to apply PirogovSinai theory (see [BK95], where the methods developed here are used to prove the existence of staggered charge order in the narrow band extended Hubbard model)

\section{Definition of the Model, Statements of Results}

21 Assumptions on the classical model We start this section by stating the precise assumptions for the classical model We consider a classical spin system with finite spin space $S=\{1, \ldots,|S|\}$, spin configurations $s: \mathbb{Z}^{d} \rightarrow S, x \mapsto s_{x}$, and finite range Hamiltonian $H^{(0)}(s)$ with translation invariant interactions, depending on a vector parameter $\mu \in \mathscr{U}$, where $\mathscr{U}$ is an open subset of $\mathbb{R}^{\prime \prime}$. We assume that $H^{(0)}(s)$ is given in the form

$$
H^{(0)}(s)=\sum_{x} \Phi_{x}(s)
$$

where $\Phi_{x}(s) \in \mathbb{R}$ depends on $s$ only via the spins $s_{y}$ for which $y \in U(x)=\{y \in$ $\left.\mathbb{Z}^{d} \mid \operatorname{dist}(x, y) \leqq R_{0}\right\}$, where $R_{0}$ is a finite number. In our notation we suppress the dependence of $H^{(0)}$ on $\Phi_{x}$ and $\mu$.

As usual, a configuration $g$ which minimises the Hamiltonian (2.1) is called a ground state configuration. For the purpose of this paper, we will assume that the number of ground states of the Hamiltonian (2.1) is finite, and that all of them are periodic More precisely, we will assume that there is a finite number of periodic configurations $g^{(1)}, \ldots, g^{(r)}$, with (specific) energies

$$
e_{m}=e_{m}(\mu)=\lim _{\Lambda \rightarrow \mathbb{Z}^{d}} \frac{1}{|\Lambda|} \sum_{x \in \Lambda} \Phi_{x}\left(g^{(m)}\right),
$$

such that for each $\mu \in \mathscr{U}$, the set of ground states $G(\mu)$ is a subset of $\left\{g^{(1)}, \ldots, g^{(t)}\right\}$. Obviously, $G(\mu)$ is given by those configurations $g^{(m)}$ for which $e_{m}(\mu)$ is equal to the "ground state energy"

$$
e_{0}=e_{0}(\mu)=\min _{m} e_{m}(\mu)
$$

Note that we may assume, without loss of generality, that $\Phi_{x}\left(g^{(m)}\right)$ is independent of the point $x$ for all ground state configurations $g^{(m)}$, because this condition can always be achieved by averaging $\Phi_{x}(s)$ in (2.1) over the minimal common period of $g^{(1)}, \ldots, g^{(r)}$

Our goal will be to prove that the low temperature phase diagram of the quantum model is a small perturbation of the classical ground state diagram provided the quantum perturbation is sufficiently small. In order to formulate and prove this statement, we need some assumptions on the structure of the ground state diagram Here we assume that for some value of $\mu_{0} \in \mathscr{U}$ all states in $\left\{g^{(1)}, ., g^{(r)}\right\}$ are ground states,

$$
e_{m}\left(\mu_{0}\right)=e_{0}\left(\mu_{0}\right) \text { for all } m=1, \ldots, r,
$$

that $e_{m}(\mu)$ are $C^{1}$ functions in $\mathscr{U}$, and that the matrix of derivatives

$$
E=\left(\frac{\partial e_{m}(\mu)}{\partial \mu_{i}}\right)
$$


has rank $r-1$ for all $\mu \in \mathscr{U}$, with uniform bounds on the inverse of the corresponding submatrices. We remark that this condition implies that the zero temperature phase diagram has the usual structure of a $v-(r-1)$ dimensional coexistence surface $S_{0}$ where all states $g^{(m)}$ are ground states, $r$ different $v-(r-1)+1$ dimensional surfaces $S_{n}$ ending in $S_{0}$ where all states but the state $g^{(m)}$ are ground states, ....

Next, we formulate a suitable Peierls condition. In order to present it, we introduce, for a given configuration $s$, the notion of excited sites $x \in \mathbb{Z}^{d}$. We say that a site $x$ is in the state $g^{(m)}$ if the configuration $s$ coincides with the configuration $g^{(m)}$ on $U(x)$, i.e. on all sites $y$ for which dist $(x, y) \leqq R_{0}$; a site is excited, if it is not in any of the states $g^{(1)}, \ldots, g^{(r)}$. Given this notation, the Peierls assumption used in this paper is that there exists a constant $\gamma_{0}>0$, independent of $\mu$, such that

$$
\Phi_{x}(s) \geqq e_{0}(\mu)+\gamma_{0} \quad \text { for all excited sites } x \text { of all configurations } s \text {. }
$$

Finally, we assume that the derivatives of $\Phi_{x}$ are uniformly bounded in $\mathscr{U}$. More explicitly, we assume that there is a constant $C_{0}<\infty$, such that

$$
\left|\frac{\partial}{\partial \mu_{i}} \Phi_{x}(s)\right| \leqq C_{0}
$$

for all $i=1, \ldots, v, \mu \in \mathscr{U}, x \in \mathbb{Z}^{d}$, and all configurations $s$.

Remark. Given the assumptions stated in this subsection, standard Pirogov-Sinai theory implies that the low temperature phase diagram of the classical model has the same topological structure as the corresponding zero temperature phase diagram (see above).

2.2. Assumptions on the quantum perturbation. As pointed out in the introduction, we propose to develop a theory which allows to control low temperature quantum spin systems that are small perturbations of the classical system introduced above. We consider quantum spin systems with Hamiltonians of the form

$$
H=H^{(0)}+\lambda V,
$$

where $H^{(0)}$ is diagonal in a basis $|s\rangle=\bigotimes_{x}\left|s_{x}\right\rangle$ that may be labelled by the configurations $s=\left\{s_{x}\right\}$ of the classical spin system,

$$
H^{(0)}|s\rangle=H^{(0)}(s)|s\rangle \text {. }
$$

We assume that $V$ is of the form

$$
V=\sum_{A} V_{A}
$$

where the sum goes over connected sets $A$ and $V_{A}$ is a self-adjoint operator on $\mathscr{H}_{A}=$ $\bigotimes_{x \in A} \mathscr{H}_{x}$. In addition to translation invariance, we assume that $V_{A}$ and its derivatives, $\frac{\partial}{\partial \mu_{l}} V_{A}, i=1, \ldots, v$, are bounded operators, with a suitable decay constraint on the corresponding operator norms $\left\|V_{A}\right\|$ and $\left\|\frac{\partial}{\partial \mu_{l}} V_{A}\right\|$. In order to formulate this constraint, 
we introduce the Sobolev norm

$$
\|V\|_{\gamma}=\sum_{A . x \in A}\left(\left\|V_{A}\right\|+\sum_{i=1}^{\gamma}\left\|\frac{\partial}{\partial \mu_{l}} V_{A}\right\|\right) e^{\gamma|A|},
$$

where $|A|$ is the number of points in $A$ Given this definition, our assumption on the decay of $V$ is the assumption that

$$
\|V\|_{i_{Q}}<\infty
$$

for a sufficiently large constant $\gamma_{Q}$.

\section{Remarks.}

i) For a finite range perturbation, where $V_{A}=0$ if the diameter of $A$ exceeds the range $R_{Q}$ of the interaction, the assumption (2.12) is automatically fulfilled for arbitrary large $\gamma_{Q}<\infty$.

ii) If the quantum perturbation $V$ is of infinite range, we need that $\left\|V_{A}\right\|$ and $\left\|\frac{\partial}{\partial \mu_{l}} V_{A}\right\|$ decay exponentially in the size $|A|$ of $A$. Assuming exponential decay with a sufficiently large decay constant $\gamma$, and observing that the number of connected sets $A$ of size $s$ that contain a given point $x \in \mathbb{Z}^{d}$ is bounded by $(2 d)^{2 s}$, the condition (2 12) can be satisfied provided $\gamma>\gamma_{Q}+2 \log (2 d)$.

iii) Strictly speaking, an exponentially decaying pair potential, $V=\sum_{x, y} V_{x, y}$, where the norm $\left\|V_{x, y}\right\|$ decays exponentially with the distance between $x$ and $y$, is not in the class considered in this section because $V$ is not given as a sum over connected sets $A$ It is obvious, however, that such a potential can be rewritten in the required form, by artificially connecting the two points $x$ and $y$ by a nearest neighbor path In (2.11), this effectively replaces the size of the set $A=\{x, y\}$ by its $l_{1}$-diameter $\sum_{t}\left|x_{i}-y_{i}\right|$.

23 Finite volume states for the quantum system In order to discuss the phase diagram of the quantum spin system, we will consider suitable finite volume states $\langle\cdot\rangle_{q, \Lambda}$ which are analogues of the classical states with boundary condition $q$, where $q=1, ., r$ We first introduce, for any configuration $s$ and any finite set $A$, the vector $\left|s_{A}\right\rangle=\bigotimes_{x \in A}\left|s_{x}\right\rangle$. Given a finite set $\Lambda \subset \mathbb{Z}^{d}$, we then define suitable finite volume Hamiltonians $H_{\Lambda}^{(0)}$ and $H_{\Lambda}$ on the Hilbert space $\mathscr{H}_{\bar{\Lambda}}=\bigotimes_{x \in \bar{\Lambda}} \mathscr{H}_{x}$, where $\bar{\Lambda}=\bigcup_{x \in \Lambda} U(x)$. Namely, we introduce operators

$$
\begin{gathered}
H_{x}^{(0)}=\sum_{s_{\bar{A}}} \Phi_{x}\left(s_{\bar{\Lambda}}\right)\left|s_{\bar{\Lambda}}\right\rangle\left\langle s_{\bar{\Lambda}}\right| \\
H_{\Lambda}^{(0)}=\sum_{x \in \Lambda} H_{x}^{(0)},
\end{gathered}
$$

and

$$
H_{\Lambda}=H_{\Lambda}^{(0)}+\lambda \sum_{A \subset A} V_{A}
$$

The Hamilton operator with boundary conditions $q$ is then defined as the "partial expectation value"

$$
H_{q, \Lambda}=\left\langle g_{\partial \Lambda}^{(q)}\left|H_{\Lambda}\right| g_{\hat{\imath} \Lambda}^{(q)}\right\rangle,
$$


where $\partial \Lambda$ is the set $\bar{\Lambda} \backslash \Lambda$. More precisely, $H_{q, \Lambda}$ is an operator on $\mathscr{H}_{\Lambda}$ whose matrix elements are

$$
\left\langle s_{\Lambda}\left|H_{q, \Lambda}\right| s_{\Lambda}^{\prime}\right\rangle=\left\langle s_{\Lambda}\left|\otimes\left\langle g_{\bar{\Lambda} \backslash \Lambda}^{(q)}\left|H_{\Lambda}\right| g_{\bar{\Lambda} \backslash \Lambda}^{(q)}\right\rangle \otimes\right| s_{\Lambda}^{\prime}\right\rangle .
$$

Given the Hamiltonian with boundary conditions $q$, we introduce the quantum state $\langle\cdot\rangle_{q, \Lambda}$ as

$$
\langle\cdot\rangle_{q, \Lambda}=\frac{1}{Z_{q, \Lambda}} \operatorname{Tr}_{\mathscr{H}_{\Lambda}}\left(\cdot e^{-\beta H_{q, \Lambda}}\right),
$$

where

$$
Z_{q, \Lambda}=\operatorname{Tr}_{\mathscr{H}_{\Lambda}} e^{-\beta H_{q, \Lambda}}
$$

Note that $H_{\Lambda}^{(0)}$ and $H_{\Lambda}$ are operators on $\mathscr{H}_{\bar{\Lambda}}$, while $H_{q, \Lambda}$ and its analogues,

$$
H_{q, x}^{(0)}=\left\langle g_{\partial \Lambda}^{(q)}\left|H_{x}^{(0)}\right| g_{\partial \Lambda}^{(q)}\right\rangle
$$

and

$$
H_{q, \Lambda}^{(0)}=\left\langle g_{\partial \Lambda}^{(q)}\left|H_{\Lambda}^{(0)}\right| g_{\partial \Lambda}^{(q)}\right\rangle
$$

are operators on $\mathscr{H}_{\Lambda}$.

Remark. Following Ginibre [Gin69], it might seem more natural to implement the boundary conditions with the help of suitable projection operators $P_{\partial \Lambda}^{(q)}$. Here, this would amount to defining $P_{\partial \Lambda}^{(q)}=\left|g_{\partial \Lambda}^{(q)}\right\rangle\left\langle g_{\partial \Lambda}^{(q)}\right|$. With the help of this projection operator, one would then define

$$
Z_{q, \Lambda}=\operatorname{Tr} P_{\partial \Lambda}^{(q)} e^{-\beta H_{\Lambda}}
$$

and similarly for the finite volume states (2.18). Observing that

$$
\operatorname{Tr}_{\mathscr{H}_{\Lambda}} W e^{-\beta H_{q, \Lambda}}=\operatorname{Tr}_{\mathscr{H}_{\bar{\Lambda}}} W P_{\partial \Lambda}^{(q)} e^{-\beta H_{\Lambda}}
$$

for all operators $W$ on $\mathscr{H}_{\Lambda}$, these two implementations of boundary conditions are actually equivalent.

2.4. Statement of results. In order to state our results in the form of a theorem, we recall that a local observable is an operator which is a selfadjoint bounded operator on $\mathscr{H}_{\Lambda}$ for some finite set $\Lambda$. We also introduce, for each $x$ in $\mathbb{Z}^{d}$ and any local observable $\Psi$, the translate $t_{x}(\Psi)$. Defining finally $\Lambda(L)$ as the box

$$
\Lambda(L)=\left\{x \in \mathbb{Z}^{d}|| x_{i} \mid \leqq L \text { for all } i=1, \ldots, d\right\},
$$

our main results are stated in the following two theorems.

Theorem 2.1. Let $d \geqq 2$ and let $H^{(0)}$ be a Hamiltonian obeying the assumptions of Sect. 2.1. Then there are constants $0<\beta_{0}<\infty$ and $0<\gamma_{Q}<\infty$, such that for all quantum perturbations $V$ obeying the assumptions of Sect. 2.2, all $\beta \geqq \beta_{0}$ and all $\lambda \in \mathbb{C}$ with

$$
|\lambda| \leqq \lambda_{0}:=\frac{1}{e \beta_{0} \mid\|V\|_{\gamma_{Q}}}
$$


there are constants $\xi_{q}$ and continuously differentiable functions $f_{q}(\mu), q=1, \quad, r$, such that the following statements hold true whenever

$$
a_{q}(\beta, \lambda, \mu):=\operatorname{Re} f_{q}(\mu)-\min _{m} \operatorname{Re} f_{m}(\mu)=0 .
$$
to $f_{q}$.

i) The infinite volume free energy corresponding to $Z_{q, \Lambda(L)}$ exists and is equal

$$
f_{q}=-\frac{1}{\beta} \lim _{L \rightarrow \infty} \frac{1}{|\Lambda(L)|} \log Z_{q, \Lambda(L)}
$$

ii) The infinite volume limit

$$
\langle\Psi\rangle_{q}=\lim _{L \rightarrow \infty}\langle\Psi\rangle_{q, \Lambda(L)}
$$

exists for all local observables $\Psi$ that

iii) For all local observables $\Psi$ and $\Phi$, there exists a constant $C_{\Psi, \Phi}<\infty$, such

$$
\left|\left\langle\Psi t_{x}(\Phi)\right\rangle_{q}-\langle\Psi\rangle_{q}\left\langle t_{x}(\Phi)\right\rangle_{q}\right| \leqq C_{\Psi, \Phi} e^{-|x| / \zeta_{q}}
$$

iv) The projection operators

$$
P_{U(x)}^{(q)}=\left|g_{U(x)}^{(q)}\right\rangle\left\langle g_{U(x)}^{(q)}\right|
$$

onto the "classical states" $g_{U(x)}^{(q)}$ obey the bounds

$$
\left|\left\langle P_{U(x)}^{(q)}\right\rangle_{q}-1\right|<\frac{1}{2}
$$

and

$$
\left|\left\langle P_{U(x)}^{(m)}\right\rangle_{q}\right|<\frac{1}{2}
$$

for all $m \neq q$

v) There exists a point $\tilde{\mu}_{0} \in \mathcal{U}$ such that $a_{m}\left(\tilde{\mu}_{0}\right)=0$ for all $m=1, \ldots, r$ For all $\mu \in \mathscr{U}$, the matrix of derivatives

$$
F=\left(\frac{\partial \operatorname{Re} f_{m}(\mu)}{\partial \mu_{i}}\right)
$$

has rank $r-1$, and the inverse of the corresponding submatrix is uniformly bounded in $\mathscr{U}$

Remarks

i) Following the usual terminology of Pirogov-Sinai theory, we call a phase with $a_{q}=0$ stable By the inverse function theorem, statement $\mathrm{v}$ ) of the theorem implies that the phase diagram of the quantum system has the same structure as the zero temperature phase diagram of the classical sytem, with a $v-(r-1)$ dimensional coexistence surface $\tilde{S}_{0}$ where all states are stable, $r$ different $v-(r-1)+1$ dimensional surfaces $\tilde{S}_{n}$ ending in $\tilde{S}_{0}$ where all states but the state $m$ are stable,....

ii) Choosing $\beta$ sufficiently large and $\lambda$ sufficiently small, the bounds (2.29) and (2.30) can be made arbitrarily sharp. In this sense, the quantum states $\langle\cdot\rangle_{q}$ are small perturbations of the corresponding classical states whenever $q$ is stable. 
iii) While Theorem 2.1 is stated (and proven) for general complex $\lambda$, the physical situation corresponds, of course, to real values of $\lambda$, as required by the selfadjointness of the Hamiltonian $H$. As we will see in Sect. 5, the "meta-stable free energies" $f_{q}$ are real in this case, making the real part in (2.24) and (2.31) superfluous.

In order to state the next theorem, we define states with periodic boundary conditons on $\Lambda(L)$. To this end, we consider the torus $\Lambda_{\text {per }}(L)=(\mathbb{Z} /(2 L+1) \mathbb{Z})^{d}$ and the corresponding Hamiltonian

$$
H_{\mathrm{per}, \Lambda(L)}=\sum_{x \in \Lambda_{\mathrm{per}}(L)} H_{x}^{(0)}+\lambda \sum_{A \subset \Lambda_{\mathrm{per}}(L)} V_{A},
$$

where the second sum goes over all subsets $A \subset \Lambda_{\text {per }}(L)$ which do not wind around the torus $\Lambda_{\text {per }}(L)$. With these definitions, we then introduce the quantum state with periodic boundary conditions as

$$
\langle\cdot\rangle_{\mathrm{per}, \Lambda(L)}=\frac{1}{Z_{\mathrm{per}, \Lambda(L)}} \operatorname{Tr}_{\mathscr{H}_{\Lambda(L)}}\left(\cdot e^{-\beta H_{\mathrm{per}, \Lambda(L)}}\right),
$$

where

$$
Z_{\mathrm{per}, \Lambda(L)}=\operatorname{Tr}_{\mathscr{H}_{\Lambda(L)}} e^{-\beta H_{\mathrm{per}, \Lambda(L)}} .
$$

Theorem 2.2. Let $H^{(0)}, V, \beta$ and $\lambda$ as in Theorem 2.1. Assume in addition that $\lambda$ is real. Then the infinite volume state with periodic boundary conditions,

$$
\langle\Psi\rangle_{\mathrm{per}}=\lim _{L \rightarrow \infty}\langle\Psi\rangle_{\mathrm{per}, \Lambda(L)}
$$

exists for all local observables $\Psi$, and is a convex combination (with equal weights) of the stable states,

$$
\langle\Psi\rangle_{\mathrm{per}}=\sum_{q \in Q(\mu)} \frac{1}{|Q(\mu)|}\langle\Psi\rangle_{q}
$$

Here

$$
Q(\mu)=\left\{q \in\{1, \ldots, r\} \mid a_{q}(\mu)=0\right\}
$$

\section{Derivation of the Contour Representation}

As explained in the introduction, we start with the Duhamel expansion for the transfer matrix. In this section, we will consider a fixed finite volume $\Lambda=\Lambda(L)=\{x \in$ $\mathbb{Z}^{d}|| x_{i} \mid \leqq L$ for all $\left.i=1, \ldots, d\right\}$, and a fixed value $q \in\{1, \ldots, r\}$ for the boundary condition; further, we are not explicitly specifying this in our notation. Introducing the transfer matrices

$$
T^{(0)}=e^{-\tilde{\beta} H_{q, \Lambda}^{(0)}}
$$

and

$$
T=e^{-\tilde{\beta} H_{q, \Lambda}},
$$

we rewrite the partition function $Z_{q, \Lambda}$ as

$$
Z_{q, \Lambda}=\operatorname{Tr} T^{M},
$$


where $\tilde{\beta}$ and $M \in \mathbb{N}$ are related to the inverse temperature $\beta$ by the equality

$$
\beta=M \tilde{\beta}
$$

The Duhamel expansion (or Dyson series) for the operator $T$ yields

$$
T=\sum_{\mathbf{n}}\left[\prod_{A \in \mathscr{Q}_{0}} \frac{(-\lambda)^{n_{A}}}{n_{A} !} \int_{0}^{\tilde{\beta}} d \tau_{A}^{1} \quad d \tau_{A}^{n_{4}}\right] T(\tau, \mathbf{n})
$$

Here, $\mathscr{A}_{0}$ is the family of all sets $A$ contributing to the sum (215), $\mathbf{n}$ is a multiindex $\mathbf{n}: \mathscr{A}_{0} \rightarrow\{0,1, \ldots$,$\} with finite n=\sum_{A \in \mathscr{A}_{0}} n_{A}, \tau=\left\{\tau_{A}^{1}, \ldots, \tau_{A}^{n_{A}}, A \in \mathscr{A}_{0}\right\} \in[0, \tilde{\beta}]^{n}$, and the operator $T(\tau, \mathbf{n})$ is obtained from $T^{(0)}$ by "inserting" the operator $V_{A}$ at the times $\tau_{A}^{1}, \quad, \tau_{A}^{n_{A}}$ Formally, it can be defined as follows For a given $\mathbf{n}$ and $\tau$, let $\operatorname{supp} \mathbf{n} \equiv \mathscr{A}=\left\{A_{1}, \quad, A_{k}\right\}$ be the set of all $A \in \mathscr{A}_{0}$ with $n_{A} \neq 0, n_{i}=n_{A_{i}}$, and $V_{i}=V_{A_{i}}$ Let

$$
\left(s_{1}, \ldots, s_{n}\right)=\pi\left(\tau_{A_{1}}^{1}, \ldots, \tau_{A_{1}}^{n_{1}}, \ldots, \tau_{A_{k}}^{1}, \ldots, \tau_{A_{k}}^{n_{k}}\right)
$$

be a permutation of the times $\tau$ such that $s_{1} \leqq s_{2} \leqq \ldots \leqq s_{n}$, and set

$$
\left(\tilde{V}_{1}, \ldots, \tilde{V}_{n}\right)=\pi\left(V_{1}, \ldots, V_{1}, \quad, V_{k}, \ldots, V_{k}\right),
$$

where on the right-hand side each $V_{i}$ appears exactly $n_{l}$ times. Then $T(\tau, \mathbf{n})$ is defined by

$$
T(\tau, \mathbf{n})=e^{-s_{1} H_{q}^{(0)}} \tilde{V}_{1} e^{-\left(s_{2}-s_{1}\right) H_{q}^{(0)}} \tilde{V}_{2} \quad e^{-\left(s_{n}-s_{n-1}\right) H_{q}^{(0)}} \tilde{V}_{n} e^{-\left(\tilde{\beta}-s_{n}\right) H_{q 1}^{(0)}} .
$$

Next, we resum (3.5) to obtain the expansion

$$
T=\sum_{B \subset A} T(B),
$$

where

$$
T(B)=\sum_{\substack{\mathscr{A}=\left\{A_{1}, A_{k}\right\} \\ \cup \\ A_{1}=B}} \tilde{T}(\mathscr{A}),
$$

with

$$
\tilde{T}(\mathscr{A})=\sum_{\mathbf{n}: \operatorname{supp} \mathbf{n}=\mathscr{A}}\left[\prod_{A \in \mathscr{A}} \frac{(-\lambda)^{n_{4}}}{n_{A} !} \int_{0}^{\tilde{\beta}} d \tau_{A}^{1} d \tau_{A}^{n_{A}}\right] T(\tau, \mathbf{n}) .
$$

Before continuing with the expansion for the partition function as sketched in the introduction, we discuss the factorization properties of the operator $T(B)$ Given a subset $\Lambda^{\prime}$ of $\Lambda$, we introduce the operators $T_{A^{\prime}}(\tau, \mathbf{n}), \tilde{T}_{A^{\prime}}(\mathscr{A})$, and $T_{A^{\prime}}(B)$ that are obtained from $T(\tau, \mathbf{n}), \hat{T}(\mathscr{A})$, and $T(B)$, respectively, by replacing $H_{q, A}^{(0)}=\sum_{x \in A} H_{q, r}^{(0)}$ by the operator $\sum_{x \in \Lambda^{\prime}} H_{q, x}^{(0)}$. Using the fact that

$$
\left[H_{q, x}^{(0)}, H_{q, v}^{(0)}\right]=0 \quad \text { for all } x, y \in \Lambda,
$$

while

$$
\left[H_{q, x}^{(0)}, V_{A}\right]=0 \quad \text { if } \operatorname{dist}(x, A)>R_{0},
$$


one immediately obtains that

$$
T(B)=T_{\Lambda \backslash \bar{B}}^{(0)} T_{\bar{B}}(B)=T_{\bar{B}}(B) T_{\Lambda \backslash \bar{B}}^{(0)},
$$

where $\bar{B}$ is the set

$$
\bar{B}=\left\{x \in \Lambda \mid \operatorname{dist}(x, B) \leqq R_{0}\right\} .
$$

Let us now consider a set $B$ that can be decomposed as $B=B_{1} \cup B_{2}$, with $\bar{B}_{1} \cap$ $\bar{B}_{2}=\emptyset$. Then

$$
T_{\bar{B}}(B)=T_{\bar{B}_{1}}\left(B_{1}\right) T_{\bar{B}_{2}}\left(B_{2}\right)=T_{\bar{B}_{2}}\left(B_{2}\right) T_{\bar{B}_{1}}\left(B_{1}\right),
$$

due to $(3.10),(3.11)$, and the fact that

$$
\left[V_{A}, V_{A^{\prime}}\right]=0 \quad \text { if } A \cap A^{\prime}=\emptyset .
$$

For $B \subset A$, we therefore get the decompositions

$$
T_{\bar{B}}(B)=\prod_{i=1}^{k} T_{\bar{B}_{l}}\left(B_{i}\right)
$$

and

$$
T(B)=T_{\Lambda \backslash \bar{B}}^{(0)} \prod_{i=1}^{k} T_{\bar{B}_{t}}\left(B_{i}\right)
$$

provided $B=\bigcup_{i=1}^{k} B_{i}$, where $\bar{B}_{1}, \ldots, \bar{B}_{k}$ are pairwise disjoint.

Deviating a little bit from the strategy explained in the introduction, we further expand the transfer matrix $T$. Using (3.17), we observe that

$$
\begin{aligned}
& \left\langle s_{B}\left|\otimes\left\langle s_{\Lambda \backslash B}|T(B)| s_{\Lambda \backslash B}^{\prime}\right\rangle \otimes\right| s_{B}^{\prime}\right\rangle \\
& \quad=\delta_{s_{\Lambda \backslash B}, s_{\Lambda \backslash B}^{\prime}} e^{-\tilde{\beta} \sum_{x \in A \backslash \bar{B}} \Phi_{x}\left(s_{U(x)}\right)}\left\langle s_{B}\left|\otimes\left\langle s_{\Lambda \backslash B}\left|T_{\bar{B}}(B)\right| s_{\Lambda \backslash B}^{\prime}\right\rangle \otimes\right| s_{B}^{\prime}\right\rangle .
\end{aligned}
$$

Introducing thus the operator $T_{B}\left(s_{\overline{\partial B}}\right)$ on $\mathscr{H}_{B}$ as the partial expectation value

$$
T_{B}\left(s_{\overline{\partial B}}\right)=\left\langle s_{\Lambda \backslash B}\left|T_{\bar{B}}(B)\right| s_{\Lambda \backslash B}\right\rangle,
$$

we get

$$
T(B)=\sum_{s_{\Lambda \backslash B}} e^{-\tilde{\beta} \sum_{x \in \Lambda \backslash \bar{B}} \Phi_{x}\left(s_{U(x)}\right)}\left(\left|s_{\Lambda \backslash B}\right\rangle\left\langle s_{\Lambda \backslash B}\right| \otimes T_{B}\left(s_{\overline{\partial B}}\right)\right)
$$

As before, $U(x)=\left\{y \in \mathbb{Z}^{d} \mid \operatorname{dist}(x, y) \leqq R_{0}\right\}$, while $\overline{\partial B}$ is the set

$$
\overline{\partial B}=\left\{x \notin B \mid \operatorname{dist}(x, B) \leqq 2 R_{0}\right\} .
$$

Considering "configurations" $\Sigma=\left(B, s_{\Lambda \backslash B}\right)$ on $\Lambda$ specifying the set $B$ as well as the configuration $s_{\Lambda \backslash B}$ outside it, we can combine (3.7) and (3.20) to get the expansion

$$
T=\sum_{\Sigma} K(\Sigma)
$$

Here the operators $K(\Sigma)$ (on $\mathscr{H}_{\Lambda}$ ) are defined by

$$
K(\Sigma) \equiv K\left(B, s_{\Lambda \backslash B}\right)=e^{-\tilde{\beta} \sum_{x \in \Lambda \backslash \bar{B}} \Phi_{x}\left(s_{U(x)}\right)}\left(\left|s_{\Lambda \backslash B}\right\rangle\left\langle s_{\Lambda \backslash B}\right| \otimes T_{B}\left(s_{\overline{\partial B}}\right)\right) .
$$


Remarks.

i) The operator $T_{B}\left(s_{\vec{C} B}\right)$ inherits from $T_{\bar{B}}(B)$ the factorization property (3.16). Namely,

$$
T_{B}\left(s_{\vec{c} B}\right)=\bigotimes_{i=1}^{n} T_{B_{\imath}}\left(s_{\vec{\imath} B_{l}}\right)
$$

provided $B=\bigcup_{i=1}^{n} B_{l}$ with $\bar{B}_{1}, \ldots, \bar{B}_{n}$ pairwise disjoint.

ii) For $x$ and $B$ near to the boundary of $A$, the spin configurations $s_{U(x)}$ and $s_{\bar{C} B}$ appearing in $\Phi_{x}$ and $T_{B}\left(s_{\bar{C} B}\right)$ involve spins $s_{y}$, with $y \notin \Lambda$. A more precise notation would therefore involve the spin configuration $s_{\Lambda \backslash B} \cup g_{\partial A}^{(q)}$ restricted to the sets $U(x)$ and $\overline{\partial B}$, respectively

Next, we combine the representation (3.22) for the transfer matrix $T$ with the formula (3.3) to rewrite $Z_{q, \Lambda}$ as

$$
Z_{q, \Lambda}=\sum_{\Sigma_{1},, \Sigma_{M}} w\left(\Sigma_{1}, ., \Sigma_{M}\right)
$$

with the weights

$$
w\left(\Sigma_{1}, \ldots, \Sigma_{M}\right)=\operatorname{Tr}_{\mathscr{H}_{1}} \prod_{t=1}^{M} K\left(\Sigma_{t}\right) .
$$

For a given collection of configurations $\Sigma_{1}, ., \Sigma_{M}, \Sigma_{t}=\left(B^{(t)}, s_{\Lambda \backslash B^{(t)}}^{(t)}\right)$, on "time slices" $t=1, \ldots, M$, we now assign a variable $\sigma_{(r, t)} \in \bar{S}=\{0,1, \ldots,|S|\}$ to each point in "space-time" lattice

$$
\mathbb{L}=\mathbb{Z}^{d} \times\{1, \quad, M\}
$$

by defining

$$
\sigma_{(r, t)}= \begin{cases}g_{x}^{(q)} & \text { if } x \notin \Lambda, \\ s_{x}^{(t)} & \text { if } x \in \Lambda \backslash B^{(t)} \\ 0 & \text { if } x \in B^{(t)}\end{cases}
$$

Considering elementary cubes, i.e. the closed unit cubes $C(x, t)$ with center $(x, t)$ in

$$
\mathbb{L}_{\Lambda}=\Lambda \times\{1, \ldots, M\}
$$

we say that a cube $C(x, t)$ is in the ground state $m$, if the configuration $\sigma_{(y, t)}$ coincides with the configuration $g^{(m)}$ on all points $y \in U(x)$. Otherwise, the cube $C(x, t)$ is called an excited cube Note that a cube $C(x, t)$ may be excited for two reasons (possibly both): either the $R_{0}$-neighborhood $U(x)$ of $x$ contains a point $y \in B^{(t)}$, corresponding to the insertion of some operator $V_{A}$ with $y \in A$, i.e. due to a quantum excitation, or it contains a point $y$ for which the classical variable $s_{y}^{(t)}$ differs from the ground state value $g_{y}^{(m)}$, which corresponds to a classical excitation. Note also that a configuration where two successive cubes $C(x, t)$ and $C(x, t+1)$ are in a classical state, $\sigma_{(x, t)}=s_{x}^{(t)}$ and $\sigma_{(x, t+1)}=s_{x}^{(t+1)}$, has weight zero unless $\sigma_{(x, t)}=\sigma_{(x, t+1)}$; indeed, otherwise one has $\left\langle s_{x}^{(t)}\left|K\left(\Sigma_{t}\right) K\left(\Sigma_{t+1}\right)\right| s_{x}^{(t+1)}\right\rangle=0$. This is true also for $t=M$ once we identify $t=M+1$ with $t=1$ (periodic boundary conditions on $\mathbb{L}$ ). 
Recalling the relation (3.23), we now extract a factor $e^{-\tilde{\beta} \Phi_{x}\left(g^{(m)}\right)} \equiv e^{-\tilde{\beta} e_{m}}$ for each cube $C$ in the ground state $m$, leading to an overall factor of

$$
e^{-\tilde{\beta} \sum_{m} e_{m}\left|W_{m}\right|},
$$

where $\left|W_{m}\right|$ is the number of cubes in the ground state $m$. Considering, on the other hand, the union $D$ of all excited cubes, we assign a label $\alpha_{D}(F)$ to all elementary faces $F$ in the boundary of $D$, by defining $\alpha_{D}(F)=m$ if $F$ is a common face for a cube $C_{D}$ in $D$ and a cube $C_{m}$ outside $D$ in the ground state $m, F=C_{D} \cap C_{m}$. Defining the reduced weight $\omega\left(\sigma_{\mathbb{L}}\right)$ by

$$
w\left(\Sigma_{1}, \ldots, \Sigma_{M}\right)=e^{-\tilde{\beta} \sum_{m} e_{m}\left|W_{m}\right|} \omega\left(\sigma_{\mathbb{L}}\right),
$$

we observe that $\omega\left(\sigma_{\mathbb{L}}\right)$ depends only on the configuration $\sigma_{D}$ and the label $\alpha_{D}$,

$$
\omega\left(\sigma_{\mathbb{L}}\right)=\omega\left(\sigma_{D}, \alpha_{D}\right)
$$

(The configuration outside $D$ is entirely determined by the labels $\alpha_{D}$.) The weight $\omega\left(\sigma_{D}, \alpha_{D}\right)$ inherits from (3.24) the factorization property

$$
\omega\left(\sigma_{D}, \alpha_{D}\right)=\prod_{i=1}^{n} \omega\left(\sigma_{D_{\imath}}, \alpha_{D_{t}}\right)
$$

Here $D_{1}, \ldots, D_{n}$ are the connected components of $D$.

At this point, the rest is standard. One considers the sets

$$
\mathbb{T}_{\Lambda}=\bigcup_{(x, t) \in \mathbb{L}_{\Lambda}} C(x, t)
$$

and

$$
\mathbb{T}=\bigcup_{(x, t) \in \mathbb{L}_{\infty}} C(x, t),
$$

imposing periodic boundary conditions in the "time direction," and defines a (labeled) contour $Y$ as a pair $(\operatorname{supp} Y, \alpha)$, where $\operatorname{supp} Y \subset \mathbb{T}_{\Lambda}$ is a connected union of closed unit cubes with centers in $\mathbb{L}_{\Lambda}$ (considered as a subset of $\mathbb{T}$ ), while $\alpha$ is an assignment of a label $\alpha(F)$ to faces of $\partial \operatorname{supp} Y$ which is constant on the boundary of all connected components of $\mathbb{T} \backslash \operatorname{supp} Y$.

The contours $Y_{1}, \ldots, Y_{n}$ corresponding to a configuration $\sigma_{\mathbb{L}_{A}}$ are then defined by taking the connected components of the set $D$ of excited cubes in $\mathbb{T}_{\Lambda}$ for their supports supp $Y_{1}, \ldots, \operatorname{supp} Y_{n}$ and by taking the labels $m$ of the ground states for the cubes $C$ in $\mathbb{T}_{\Lambda} \backslash \operatorname{supp} Y_{i}$ that touch the face $F$, see above, for the corresponding labels $\alpha_{i}(F)$.

Resumming over all configurations in (3.25) that lead to the same set of contours, and taking into account the factorization property (3.33), it is an easy exercise to show that the resulting weight factors into a product of contour activities $\rho\left(Y_{i}\right)$ and ground state terms $e^{-\tilde{\beta} e_{m}\left|W_{m}\right|}$. This yields the representation

$$
Z_{q, \Lambda}=\sum_{\left\{Y_{1},, Y_{n}\right\}} \prod_{i} \rho\left(Y_{i}\right) \prod_{m} e^{-\tilde{\beta} e_{m}\left|W_{m}\right|},
$$


which is exactly of the same form as the contour representation of a classical spin system. We can therefore apply standard Pirogov-Sinai theory to analyze the quantum spin system considered here, provided we can verify its basic assumption - the Peierls condition. Namely, we should prove a bound of the form

$$
|\rho(Y)| \leqq e^{-\hat{y}|Y|} e^{-\tilde{\beta} e_{0}|Y|}
$$

where $\gamma$ is a sufficiently large constant, $e_{0}=\min _{m} e_{m}$, and $|Y|$ is the number of elementary cubes in supp $Y$ This will be done in the next section

Remark. The weights $\rho(Y)$ are in general complex, even if the coupling constant $\lambda$ is real. Observing that the operators $K\left(\Sigma_{t}\right)$ in (3.26) are selfadjoint for real $\lambda$, we have, however, that

$$
w\left(\Sigma_{1}, \ldots, \Sigma_{M}\right)^{*}=w\left(\Sigma_{M}, \quad, \Sigma_{1}\right)
$$

Considering two contours $Y$ and $Y^{*}$ which can be obtained from each other by a reflection at a constant time plane, we therefore get

$$
\rho(Y)^{*}=\rho\left(Y^{*}\right)
$$

provided $\lambda \in \mathbb{R}$.

\section{Exponential Decay for Contour Activities}

We first give an explicit expression for the weight $\rho(Y)$ Combining (3.25), (3.31), (3 32), and (3.33) we have

$$
Z_{q, \Lambda}=\sum_{\Sigma_{1},, \Sigma_{M}} e^{-\tilde{\beta} \sum_{m} e_{m}\left|W_{m}\right|} \prod_{l=1}^{n} \omega\left(\sigma_{D_{l}}, \alpha_{D_{l}}\right),
$$

where $D_{1}, \quad, D_{n}$ are the connected components of the union $D$ of all excited cubes corresponding to $\Sigma_{1}, \ldots, \Sigma_{M}$. This expression is equivalent to (3.36) once we take for a contour $Y \equiv\left(D, \alpha_{D}\right)$ the weight

$$
\rho(Y)=\sum_{\sigma_{D} \rightarrow Y} \omega\left(\sigma_{D}, \alpha_{D}\right)
$$

Here the sum is over all configurations $\sigma_{D}$ consistent with the contour $Y$, i.e. over all configurations $\sigma_{D}$ on $D=\operatorname{supp} Y$ that, if extended outside supp $Y$ by appropriate ground states determined by the labels $\alpha_{D}$, yield the contour $Y$

Propositon 4.1. Let $\lambda \in \mathbb{R}, \tilde{\beta}>0$, and $\gamma_{Q} \geqq 1$ be such that, for all $x \in \mathbb{Z}^{d}$,

$$
(e-1) \tilde{\beta}|\lambda| \sum_{\substack{A \in \sim_{0} \\ r \in A}}\left\|V_{A}\right\| e^{\gamma_{Q}|A|} \leqq 1 .
$$

Then

$$
|\rho(Y)| \leqq e^{-\left(\tilde{\beta} e_{0}+\hat{\imath}\right)|Y|}
$$

where

$$
\gamma=\min \left\{\tilde{\beta} \gamma_{0}, R_{0}^{-d}\left(\gamma_{Q}-1\right)\right\}-\log (2|S|)
$$

The proof of the proposition relies on the following lemma. 
Lemma 4.2. Let $B \subset A$, and let $s_{B}, \tilde{s}_{B}$ and $s_{\overline{\partial B}}$ be arbitrary classical configurations on $B$ and $\overline{\partial B}=\left\{x \notin B \mid \operatorname{dist}(x, B) \leqq 2 R_{0}\right\}$, respectively. Let $\gamma_{Q}, \tilde{\beta}$ and $\lambda$ be as in Proposition 4.1. Then

$$
\left|\left\langle s_{B}\left|T_{B}\left(s_{\overline{\partial B}}\right)\right| \tilde{s}_{B}\right\rangle\right| \leqq e^{-\tilde{\beta} e_{0}|\bar{B}|} e^{-\left(\gamma_{Q}-1\right)|B|}
$$

Proof of Lemma 4.2. Let $s_{\Lambda}$ be an arbitrary extension of the configuration $s_{B \cup \overline{\partial B}}$ to the full set $\Lambda$, and let $\tilde{s}_{\Lambda}$ be the configuration which agrees with $s_{\Lambda}$ on $\Lambda \backslash B$, and with $\tilde{s}_{B}$ on $B$. Then

$$
\left|\left\langle s_{B}\left|T_{B}\left(s_{\overline{\partial B}}\right)\right| \tilde{s}_{B}\right\rangle\right|=\left|\left\langle s_{\Lambda}\left|T_{\bar{B}}(B)\right| \tilde{s}_{\Lambda}\right\rangle\right| \leqq\left\|T_{\bar{B}}(B)\right\|
$$

where, in agreement with (3.8) and (3.9), the operator $T_{\bar{B}}(B)$ is defined by

$$
T_{\bar{B}}(B)=\sum_{\substack{\mathscr{A}=\left\{A_{1}, A_{k}\right\} \\ A_{i} \in \mathscr{A}_{0}, \cup_{j} A_{j}=B}} \tilde{T}_{\bar{B}}(\mathscr{A})
$$

and

$$
\tilde{T}_{\bar{B}}(\mathscr{A})=\sum_{\mathbf{n}: \operatorname{supp} \mathbf{n}=\mathscr{A}}\left[\prod_{A \in \mathscr{A}} \frac{(-\lambda)^{n_{A}}}{n_{A} !} \int_{0}^{\tilde{\beta}} d \tau_{A}^{1} \cdots d \tau_{A}^{n_{A}}\right] T_{\bar{B}}(\tau, \mathbf{n}) .
$$

The time-ordered operator $T_{\bar{B}}(\tau, \mathbf{n})$ is defined as in (3.6), with the Hamiltonian $\sum_{x \in \bar{B}} H_{q, x}^{(0)}$ replacing $H_{q, \Lambda}^{(0)}$. Observing that for all $s>0$,

$$
\left\|e^{-s \sum_{x \in \bar{B}} H_{q, x}^{(0)}}\right\|=e^{-s|\bar{B}| e_{0}}
$$

we now bound

$$
\left\|T_{\bar{B}}(\tau, \mathbf{n})\right\| \leqq e^{-\tilde{\beta}|\bar{B}| e_{0}} \prod_{A \in \mathscr{A}}\left\|V_{A}\right\|^{n_{A}}
$$

Combining (4.7)-(4.9) with the bound (4.11) and the fact that the assumption (4.3) implies that $\tilde{\beta}|\lambda|\left\|V_{A}\right\| \leqq 1$ for all $A \in \mathscr{A}_{0}$, we obtain

$$
\begin{aligned}
\left|\left\langle s_{B}\left|T_{B}\left(s_{\overline{\partial B}}\right)\right| \tilde{s}_{B}\right\rangle\right| \mid & \leqq e^{-\tilde{\beta} e_{0}|\bar{B}|} \sum_{\substack{A=\left\{A_{1},, A_{k}\right\} \\
A_{i} \in \mathscr{A}_{0}, \cup_{j} A_{j}=B}} \prod_{A \in \mathscr{A}}\left(\sum_{n_{A}=1}^{\infty} \frac{(\tilde{\beta}|\lambda|)^{n_{A}}}{n_{A} !}\left\|V_{A}\right\|^{n_{A}}\right) \\
& \leqq e^{-\tilde{\beta} e_{0}|\bar{B}|} \sum_{\substack{\mathscr{A}=\left\{A_{1},, A_{k}\right\} \\
A_{i} \in \mathscr{A}_{0}, \cup_{j} A_{j}=B}} \prod_{A \in \mathscr{A}}\left((e-1) \tilde{\beta}|\lambda|\left\|V_{A}\right\|\right) \\
& \leqq e^{-\tilde{\beta} e_{0}|\bar{B}|} e^{-\gamma_{Q}|B|} \sum_{\substack{\mathscr{A}=\left\{A_{1}, A_{i} \in \mathscr{A}_{0}, \cup_{j} A_{j}=B\right.}} \prod_{A \in \mathscr{A}}\left((e-1) \tilde{\beta}|\lambda|\left\|V_{A}\right\| e^{\gamma_{Q}|A|}\right) .
\end{aligned}
$$


We proceed with the bound

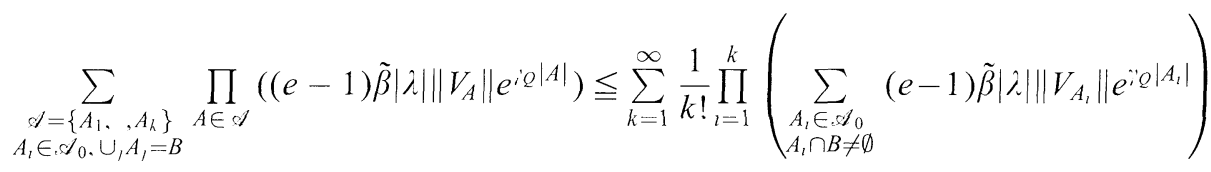

$$
\begin{aligned}
& \leqq \sum_{k=1}^{\infty} \frac{1}{k !} \prod_{i=1}^{k}\left(\sum_{\substack { x \in B \\
\begin{subarray}{c}{A_{l} \in \mathcal{A}_{0}: \\
x \in A_{l}{ x \in B \\
\begin{subarray} { c } { A _ { l } \in \mathcal { A } _ { 0 } : \\
x \in A _ { l } } }\end{subarray}}(e-1) \tilde{\beta}|\lambda||| V_{A_{l}} \| e^{\gamma^{\prime} Q}\left|A_{l}\right|\right) \\
& \leqq \sum_{k=1}^{\infty} \frac{1}{k !}|B|^{k} \leqq e^{|B|},
\end{aligned}
$$

where we have used the assumption (43) in the second to last step. Combining (4 12) and (4 13), we obtain the lemma.

Proof of Proposition 41 In order to bound the sum in (4.2), we first derive a more explicit representation for the activity $\rho(Y)$. We decompose the torus $\mathbb{T}$ and the set of excited cubes $D$ into time-slices, $\mathbb{T}=\bigcup_{t=1}^{M} \mathbb{T}^{(t)}$ and $D=\bigcup_{t=1}^{M} D^{(t)}$, and recall that $\sigma_{(x, t)}=0$ iff the cube $C(x, t)$ belongs to $B^{(t)}$, the set of sites where transitions between times $t$ and $(t+1)$ may occur. Summing over all configurations $\sigma_{D}$ consistent with the contour $Y$ then corresponds to the following three restrictions:

(1) The configuration $\sigma_{D}$ is such that the $R_{0}$-neighbourhood of each $(x, t)$ with $\sigma_{(x, t)}=0$ is included in $D$, i e $\bar{B}^{(t)} \subset D^{(t)}$ for each $t=1, ., M$.

(2) All cubes $C(x, t) \subset D$ are excited, i.e. either $\sigma_{(y, t)}=0$ for some $y \in U(x)$ or $s_{U(x)}^{(t)} \neq g_{U(x)}^{(m)}$ for all $m=1, \ldots, r$.

(3) Let $F$ be a vertical face in the boundary of $D$, let $m=\alpha_{D}(F)$ be the label of $F$, and let $\mathbb{T}^{(t)}$ be the time-slice containing $F$ Then $\sigma(x, t)=g_{x}^{(m)}$ for all $x \in D^{(t)}$ whose distance from $F$ is less then $R_{0}$.

Let us observe that since $\left|s_{\Lambda \backslash B_{1}}\right\rangle \otimes\left|s_{B_{1}}\right\rangle \equiv\left|s_{\Lambda \backslash B_{2}}\right\rangle \otimes\left|s_{B_{2}}\right\rangle$ for any $B_{1}, B_{2} \subset \Lambda$, we may write the expansion of unity $\mathbf{1}=\sum_{s_{1}}\left|s_{\Lambda}\right\rangle\left\langle s_{\Lambda}\right|$ on $\mathscr{H}_{\Lambda}$ in the form

$$
\mathbf{1}=\sum_{s_{1}}\left|s_{\Lambda \backslash B_{1}}\right\rangle \otimes\left|s_{B_{1}}\right\rangle\left\langle s_{\Lambda \backslash B_{2}}\right| \otimes\left\langle s_{B_{2}}\right| .
$$

Inserting now (3.26) with (3 31) and (3.32) into (4.2), we may use the above observation to get the expression

$$
\begin{aligned}
& \rho(Y)=\sum_{\substack{B^{(1)}, B^{(M)} \\
\bar{B}^{(I)} \subset D^{(t)}}} \sum_{s_{D^{(1)} \backslash B^{(1)}}^{(1)}, s_{D^{(M)} \backslash B^{(M)}}^{(M)}} \sum_{s_{I^{(1)}}^{(1)}, s_{I^{(M)}}^{(M)}} \prod_{t=1}^{M} e^{-\tilde{\beta} \sum_{r \in D^{(t)} \backslash \bar{B}^{(t)}} \Phi_{2}\left(s_{U^{(}(1)}^{(t)}\right)} \\
& \times\left\langle s_{D^{(t)} \backslash B^{(t)}}^{(t)} \mid s_{D^{(t)} \backslash B^{(t)}}^{(t+1)}\right\rangle\left\langle s_{B^{(t)}}^{(t)}\left|T_{B^{(t)}}\left(s_{\overline{c B}}^{(t)}\right)\right| s_{B^{(t)}}^{(t+1)}\right\rangle
\end{aligned}
$$

Here we defined $I^{(t)}=B^{(t-1)} \cap B^{(t)}$. The first two successive summations are equivalent to the sum in (4.2), while the third one implements the trace in (326). Namely, the sum over $B^{(1)}, \ldots, B^{(M)}$ in (4.15) obeys automatically the first restriction above; the second sum must respect the two others (and there is no restriction on the third 
sum). Observe that the choice of a contour $Y$ (or of several contours), together with the choice of partial configurations $s_{D^{(t)} \backslash B^{(t)}}^{(t)}, s_{I^{(t)}}^{(t)}$, at each time $t$ defines completely the configurations between each time slice.

In order to get a bound on $|\rho(Y)|$, we estimate the absolute values of the factors of the terms on the right-hand side of (4.15). Taking into account the condition (2) above and the assumption (2.6) on the classical part of the Hamiltonian, we have

$$
e^{-\tilde{\beta} \sum_{x \in D^{(t)} \bar{B}^{(t)}} \Phi_{x}\left(s_{U(x)}^{(t)}\right)} \leqq e^{-\tilde{\beta}\left(e_{0}+\gamma_{0}\right)\left|D^{(t)} \backslash \bar{B}^{(t)}\right|} .
$$

The scalar product between the two base vectors is equal to 0 or 1 . In fact, we could use it to reduce the number of terms appearing in the second sum in (4.15); however, we just bound it by one. Combined with Lemma 4.2, we finally get

$$
|\rho(Y)| \leqq \sum_{\substack{B^{(1)}, B^{(M)} \\ \bar{B}^{(t)} \subset D^{(t)}}} \prod_{t=1}^{M} \sum_{\substack{(t) \\ D^{(t)} \backslash B^{(t)}}} \sum_{I^{(t)}} e^{-\tilde{\beta}\left(e_{0}+\gamma_{0}\right)\left|D^{(t)} \backslash \bar{B}^{(t)}\right|} e^{-\tilde{\beta} e_{0}\left|\bar{B}^{(t)}\right|} e^{-\left(\gamma_{Q}-1\right)\left|B^{(t)}\right|} .
$$

The summands do not depend any more on the partial configurations $s_{D^{(t)} \backslash B^{(t)}}^{(t)}$ and $s_{I^{(t)}}^{(t)}$. Their number is bounded by

$$
\prod_{t=1}^{M}|S|^{D^{(t)} \backslash \bar{B}^{(t)}|+| I^{(t)} \mid} \leqq|S|^{|Y|} .
$$

To estimate the exponential in (4.17) we use the equality $\left|D^{(t)} \backslash \bar{B}^{(t)}\right|=\left|D^{(t)}\right|-\left|\bar{B}^{(t)}\right|$ and the bound $\left|\bar{B}^{(t)}\right| \leqq R_{0}^{d}\left|B^{(t)}\right|$. Thus $e^{-\left(\gamma_{Q}-1\right)\left|B^{(t)}\right|} \leqq e^{-\left(\gamma_{Q}-1\right) R_{0}^{-d}\left|\bar{B}^{(t)}\right|}$, and

$$
|\rho(Y)| \leqq\left[|s| e^{-\tilde{\beta} e_{0}} \max \left\{e^{-\tilde{\beta} \gamma_{0}}, e^{-\left(\gamma_{Q}-1\right) R_{0}^{-d}}\right\}\right]^{|Y|} \prod_{t=1}^{M} \sum_{\substack{B^{(t)} \\ \bar{B}^{(t)} \subset D^{(t)}}} 1 .
$$

The last sum can be easily bounded, yielding

$$
\sum_{\substack{B^{(t)} \\ \bar{B}^{(t)} \subset D^{(t)}}} 1 \leqq \sum_{B^{(t)} \subset D^{(t)}} 1=2^{\left|D^{(t)}\right|} .
$$

Combined with (4.19), this completes the proof of Proposition 4.1.

We close this section with a proposition providing the necessary bounds on derivatives:

Propositon 4.3. Let $\lambda \in \mathbb{R}, \tilde{\beta}>0$, and $\gamma_{Q} \geqq 1$ be such that, in addition to (4.3), we have

$$
e \tilde{\beta}|\lambda| \sum_{\substack{A \in s_{0} . \\ x \in A}}\left\|\frac{\partial}{\partial \mu_{i}} V_{A}\right\| e^{\gamma_{Q}|A|} \leqq 1 .
$$

Then

$$
\left|\frac{\partial}{\partial \mu_{i}} \rho(Y)\right| \leqq\left(\tilde{\beta} C_{0}+1\right)|Y| e^{-\left(\tilde{\beta} e_{0}+\gamma\right)|Y|} .
$$

Here $C_{0}$ is the constant from (2.7) and $\gamma$ is the constant defined in (4.5). 
Proof We first derive an analogue of the bound (4.6) in Lemma 4.2. To this end, we have to bound the norm of $\partial T_{\bar{B}}(\tau, \mathbf{n}) / \partial \mu_{i}$. Using the representation (3.6) in conjunction with the assumption (27) and the bound (4.10), we get

$$
\begin{aligned}
\left\|\frac{\partial}{\partial \mu_{l}} T_{\bar{B}}(\tau, \mathbf{n})\right\| \leqq & e^{-\tilde{\beta}|\bar{B}| e_{0}}\left\{\tilde{\beta} C_{0}|\bar{B}| \prod_{A \in \mathscr{S}}\left\|V_{A}\right\|^{n_{A}}\right. \\
& \left.+\sum_{J=1}^{k} n_{A_{j}}\left\|\frac{\partial V_{A_{j}}}{\partial \mu_{i}}\right\|\left\|V_{A_{j}}\right\|^{n_{i_{1}}-1} \prod_{A \in \mathscr{A} \backslash\left\{A_{j}\right\}}\left\|V_{A}\right\|^{n_{4}}\right\} .
\end{aligned}
$$

Proceeding as before, we obtain

$$
\begin{aligned}
& \left|\left\langle s_{B}\left|\frac{\partial}{\partial \mu_{i}} T_{B}\left(s_{\overline{c B}}\right)\right| \tilde{s}_{B}\right\rangle\right| \leqq e^{-\tilde{\beta} e_{0}|\bar{B}|} \sum_{\substack{\mathcal{A}=\left\{A_{1}, A_{h}\right\} \\
A_{1} \in \mathscr{A}_{0}, \cup A_{j}=B}}\left\{\tilde{\beta} C_{0}|\bar{B}| \prod_{A \in \mathscr{A}}\left((e-1) \tilde{\beta}|\lambda||| V_{A} \|\right)\right. \\
& \left.+\sum_{j=1}^{k} e \tilde{\beta}|\lambda|\left\|\frac{\partial V_{A_{1}}}{\partial \mu_{i}}\right\| \prod_{A \in \mathscr{A}}\left((e-1) \tilde{\beta}|\lambda|\left\|V_{A}\right\|\right)\right\} \\
& \leqq e^{-\tilde{\beta} e_{0}|\bar{B}|} e^{-\gamma_{Q}|B|} \sum_{k=1}^{\infty} \frac{1}{k !}|B|^{k}\left\{\tilde{\beta} C_{0}|\bar{B}|+k\right\} \\
& \leqq e^{-\tilde{\beta} e_{0}|\bar{B}|} e^{-i_{Q}|B|}\left\{\tilde{\beta} C_{0}|\bar{B}|+|B|\right\} e^{|B|} \\
& \leqq\left(\tilde{\beta} C_{0}+1\right)|\bar{B}| e^{-\tilde{\beta} e_{0}|\bar{B}|} e^{-(\tilde{)} Q-1)|B|} \text {. }
\end{aligned}
$$

Inserted into (4.15), and continuing in the same way as before, we obtain the bound (4.22)

\section{Truncated Free Energies and the Stable Phases of the Model}

While the next section will be devoted to a detailed discussion of the mean values of general local variables, here we will anticipate the fact that the state $\langle\cdot\rangle_{q}$ can be linked with the "probability" - in the ensemble (3.36) of labeled contours - that a given site $x$ is in $W_{q}$, the area outside contours and in the ground state $g^{(q)}$. To discuss the stability of phases in dependence on the "tuning" parameter $\mu$ (cf Sect. 21 ), we can thus use the standard Pirogov-Sinai theory There are only two features that are not entirely standard - the fact that the weights $\rho(Y)$ are in general complex numbers and the fact that our model is actually considered on a slab of thickness $M$ (linked with the temperature $\beta$ ) with periodic boundary conditions in the "time" direction The former was taken into account in [BI89] (also in [GKK88], but here we will base our discussion on [BI89] and later works based on it, in particular [BK90] and [BK94]) The latter is a novel feature of quantum models Even though it leads only to small modifications, it is important to realize that the metastable free energies used to determine the phase diagram will have contributions coming from contours wrapped around $\mathbb{T}_{\Lambda}$ in the "time" direction This leads to certain modifications in the definition of truncated free energies which will be described in this section

We start with some notation As usual (see also Sect 3) a contour is a pair $(\operatorname{supp} Y, \alpha)$, where $\operatorname{supp} Y \subset \mathbb{T}_{A}$ is a connected union of finitely many closed unit cubes with centers in $\mathbb{L}_{\infty}$ (we call those cubes elementary cubes in the sequel), 
while $\alpha$ is an assignment of a label $\alpha(F)$ to the faces $F$ in $\partial \operatorname{supp} Y$ which is constant on the boundary of all connected components of $\mathbb{T} \backslash \operatorname{supp} Y$. Its interior Int $Y$ is the union of all finite components of $\mathbb{T} \backslash \operatorname{supp} Y$ and $\operatorname{Int}_{m} Y$ the union of all components of Int $Y$ whose boundary is labeled by $m$. Recalling that we assumed $d \geqq 2$, we note that the set $\mathbb{T} \backslash(\operatorname{supp} Y \cup \operatorname{Int} Y)$ is a connected set, implying that the function $\alpha(\cdot)$ is constant on the boundary of the set $V(Y)=\operatorname{supp} Y \cup \operatorname{Int} Y$. We say that $Y$ is a $q$-contour, if $\alpha_{Y}=q$ on this boundary. Two contours $Y$ and $Y^{\prime}$ are called compatible or not touching if $\operatorname{supp} Y \cap \operatorname{supp} Y^{\prime}=\emptyset$, and mutually external if $V(Y) \cap V\left(Y^{\prime}\right)=\emptyset$. Given a finite set of mutually compatible contours $Y_{1}, \ldots, Y_{n}$ we say that $Y_{i}, i=1, \ldots, n$, is an external contour in $\left\{Y_{1}, \ldots, Y_{n}\right\}$, if $\operatorname{supp} Y_{i} \cap V\left(Y_{j}\right)=\emptyset$ for all $j \neq i$. Consider now a set of contours $\left\{Y_{1}, \ldots, Y_{n}\right\}$ contributing to (3.36). The contours in $\left\{Y_{1}, \ldots, Y_{n}\right\}$ are then mutually compatible, and all external contours are $q$-contours. In addition, the labels of these contours are matching in the sense that the boundary of each connected component of $\mathbb{T}_{\Lambda} \backslash\left(\operatorname{supp} Y_{1} \cup \cdots \cup \operatorname{supp} Y_{n}\right)$ has constant boundary conditions.

Given this setup, it is now standard to derive a second representation for $Z_{q, \Lambda}$ which does not involve such a matching condition. To this end, we first introduce partition functions $Z_{q}(V)$ for all volumes $V \subset \mathbb{T}_{\Lambda}$ for which $V^{c}=\mathbb{T}_{\Lambda} \backslash V$ is a (possibly empty) union of closed elementary cubes in $\mathbb{T}_{\Lambda}$. We say that $Y$ is a contour in $V$, if $V(Y) \subset V$, and call a set $\left\{Y_{1}, \ldots, Y_{n}\right\}$ of mutually compatible contours in $V$ a set of matching contours in $V$ if the boundary of each connected component of $V \backslash\left(\operatorname{supp} Y_{1} \cup \cdots \cup \operatorname{supp} Y_{n}\right)$ has constant boundary conditions. Denoting the union of those components which have boundary condition $q$ by $W_{q}$, we define

$$
Z_{q}(V)=\sum_{\left\{Y_{1},, Y_{n}\right\}} \prod_{i} \rho\left(Y_{i}\right) \prod_{m} e^{-\tilde{\beta} e_{m}\left|W_{m}\right|},
$$

where the sum runs over all sets of mutually compatible, matching contours in $V$ for which all external contours are $q$-contours. Recalling the expression (3.36) for $Z_{q, \Lambda}$, we note that the partition function (5.1) is actually equal to $Z_{q, \Lambda}$ if $V=\mathbb{T}_{\Lambda}$,

$$
Z_{q}\left(\mathbb{T}_{\Lambda}\right)=Z_{q, \Lambda}
$$

We now rewrite the partition function $Z_{q}(V)$, in a standard way, as a sum over contours without any matching condition, see e.g. [Zah84] or [BI89]. Introducing the weights

$$
K_{q}(Y):=\rho(Y) e^{\tilde{\beta} e_{q}|Y|} \prod_{m=1}^{r} \frac{Z_{m}\left(\operatorname{Int}_{m} Y\right)}{Z_{q}\left(\operatorname{Int}_{m} Y\right)},
$$

one gets

$$
Z_{q}(V)=e^{-\tilde{\beta} e_{q}|V|} \sum_{\left\{Y_{1}, Y_{n}\right\} \subset V^{0}} \prod_{k=1}^{n} K_{q}\left(Y_{k}\right),
$$

where the sum runs over all sets of mutually disjoint $q$-contours in $V$.

The weights $K_{q}(Y)$ do not, necessarily, satisfy the bound

$$
\left|K_{q}(Y)\right| \leqq \varepsilon^{|Y|}
$$

with a sufficiently small constant $\varepsilon>0$. While it turns out that such a bound can be proven for stable phases $q$, it is false for unstable phases. In order to circumvent this problem, we follow the standard strategy and construct truncated contour activities 
$K_{q}^{\prime}(Y)$, truncated partition functions

$$
Z_{q}^{\prime}(V)=e^{-\tilde{\beta} e_{q}|V|} \sum_{\left\{Y_{1}, Y_{n}\right\} \subset V^{0}} \prod_{k=1}^{n} K_{q}^{\prime}\left(Y_{k}\right),
$$

and the corresponding free energies $f_{q}$ in such a way that the weights $K_{q}^{\prime}(Y)$ satisfy the bound (5.5) and, in the same time do not differ from $K_{q}(Y)$ whenever the phase $q$ is stable, i e. whenever the real part of the free energy $f_{q}$ of the truncated model is minimal, $\operatorname{Re} f_{q}=\min _{m} \operatorname{Re} f_{m}$.

Our definition of the truncated weights $K_{q}^{\prime}$ follows closely the treatment from [BK94]. The main difference is that since the contours can have only a limited extension in the time direction, we take their "horizontal diameter" as the parameter to use in inductive definitions (and proofs) Namely, for a contour $Y$, we take the diameter $\delta(Y)$ defined as the diameter of the $d$-dimensional projection

$$
\left\{y \in \mathbb{R}^{d} \text { such that }(y, t) \in C(x, t) \text {, for some } C(x, t) \subset \operatorname{supp} Y\right\} .
$$

We define $\delta(V)$ in the same way. Notice that $\delta(\tilde{Y})<\delta(Y)$ whenever supp $\tilde{Y} \subset$ Int $(Y)$.

To introduce the weight $K_{q}^{\prime}(Y)$ in an inductive manner, assume that it has already been defined for all $q$ and all contours $Y$ with $\delta(Y)<n, n \in \mathbb{N}$, and that it obeys a bound of the form (5.5). Introduce $f_{q}^{(n-1)}$ as the free energy of a contour model with activities

$$
K^{(n-1)}\left(Y^{q}\right)= \begin{cases}K^{\prime}\left(Y^{q}\right) & \text { if } \delta\left(Y^{q}\right) \leqq n-1 \\ 0 & \text { otherwise. }\end{cases}
$$

Consider now a contour $Y$ with $\delta(Y)=n$ Since $\delta(\tilde{Y})<n$ for all contours $\tilde{Y}$ in Int $Y$, the truncated partition functions $Z_{q}^{\prime}\left(\operatorname{Int}_{m} Y\right)$ are well defined for all $q$ and $m$. Their logarithm can be controlled by a convergent cluster expansion, and $Z_{q}^{\prime}\left(\operatorname{Int}_{m} Y\right) \neq 0$ for all $q$ and $m$. We therefore may define $K_{q}^{\prime}(Y)$ for $q$-contours $Y$ with $\delta(Y)=n$ by

$$
K_{q}^{\prime}(Y)=\chi_{q}^{\prime}(Y) \rho(Y) e^{\tilde{\beta} e_{q}|Y|} \prod_{m} \frac{Z_{m}\left(\operatorname{Int}_{m} Y\right)}{Z_{q}^{\prime}\left(\operatorname{Int}_{m} Y\right)}
$$

with

$$
\chi_{q}^{\prime}(Y)=\prod_{m \neq q} \chi\left(\alpha-\tilde{\beta}\left(\operatorname{Re} f_{q}^{(n-1)}-\operatorname{Re} f_{m}^{(n-1)}\right) \delta(Y)\right) .
$$

Here $\alpha$ is a constant that will be chosen later and $\chi$ is a smoothed characteristic function We assume that $\chi$ is a $C_{1}$ function that has been defined in such a way that it obeys the conditions

$$
0 \leqq \chi(x) \leqq 1, \quad 0 \leqq \frac{d \chi}{d x} \leqq 1
$$

and

$$
\chi(x)=0 \quad \text { if } x \leqq-1 \quad \text { and } \quad \chi(x)=1 \quad \text { if } x \geqq 1 .
$$

As a final element of the construction of $K_{q}^{\prime}$, we have to establish the bound (5.5) for contours $Y$ with $\delta(Y)=n$ The proof of this fact, together with the proof of the following Lemma 5.1, follows closely [BK94]. However, since, on the one hand, the claims of Lemma 5.1 (as well as the definition of $K_{q}^{\prime}(Y)$ ) slightly differ 
from similar claims in [BK94] and, on the other hand, there is a certain number of complications in [BK94] that are not necessary for the present case, we present the proof in the appendix.

We use $f_{q}$ to denote the free energy corresponding to the partition function $Z_{q}^{\prime}(V)$

$$
f_{q}=-\frac{1}{\tilde{\beta}} \lim _{V \rightarrow \mathbb{T}} \frac{1}{|V|} \log Z_{q}^{\prime}(V),
$$

and introduce $f_{0}$ and $a_{q}$ as

$$
\begin{gathered}
f_{0}=\min _{m} \operatorname{Re} f_{m}, \\
a_{q}=\tilde{\beta}\left(\operatorname{Re} f_{q}-f_{0}\right) .
\end{gathered}
$$

Finally, we recall that volumes $V$ as well as supports of contours, supp $Y$, are unions of elementary cubes and $|Y|$ or $|\operatorname{supp} Y|$ denotes their $(d+1)$-dimensional volume. Similarly for the boundary $\partial V$ of $V$ we use $|\partial V|$ to denote its $d$-dimensional euclidean area.

Lemma 5.1. Assume that $\rho(\cdot)$ obeys the conditions (4.4) and (4.22) and let

$$
\varepsilon=e^{-\gamma+\alpha+2} \quad \text { and } \quad \bar{\alpha}=\alpha-2
$$

Then there exists a constant $\varepsilon_{0}>0$ (depending only on $d$ and $r$ ) such that the following statements hold provided $\varepsilon<\varepsilon_{0}$ and $\bar{\alpha} \geqq 1$.

i) The contour activities $K_{q}^{\prime}(Y)$ are well defined for all $Y$ and obey (5.5) and

$$
\left|\frac{\partial}{\partial \mu_{i}} K_{q}^{\prime}(Y)\right| \leqq\left(3 r \tilde{\beta} C_{0}+2\right)|V(Y)| \varepsilon^{|Y|} .
$$

ii) If $a_{q} \delta(Y) \leqq \bar{\alpha}$, then $\chi_{q}(Y)=1$ and $K_{q}(Y)=K_{q}^{\prime}(Y)$.

iii) If $a_{q} \delta(V) \leqq \bar{\alpha}$, then $Z_{q}(V)=Z_{q}^{\prime}(V)$.

iv) For all volumes $V \subset \mathbb{T}_{\Lambda}$ for which $V^{c}=\mathbb{T}_{\Lambda} \backslash V$ is a union of closed elementary cubes, one has

$$
\left|Z_{q}(V)\right| \leqq e^{-\tilde{\beta} f_{0}|V|+O(\varepsilon)|\partial V|}
$$

and

$$
\left|\frac{\partial}{\partial \mu_{i}} Z_{q}(V)\right| \leqq\left(2 \tilde{\beta} C_{0}+1\right)|V| e^{-\tilde{\beta} f_{0}|V|+O(\varepsilon)|\partial V|} .
$$

Remarks.

i) Here, as in the appendix, $O(\varepsilon)$ stands for a bound $K \varepsilon$, where $K<\infty$ is a constant that depends only on the dimension $d$ and the number of classical states $r$.

ii) For real $\lambda$, the free energy $f$ is independent of boundary conditions and can be expressed as

$$
f=-\frac{1}{\beta} \lim _{\Lambda \nearrow \mathbb{Z}^{d}} \frac{1}{|\Lambda|} \log \operatorname{Tr} \mathscr{H}_{\Lambda} e^{-\beta H_{q, \Lambda}}=-\frac{1}{\beta} \lim _{\Lambda \nearrow \mathbb{Z}^{d}} \frac{1}{|\Lambda|} \log Z_{q, \Lambda} .
$$

Since $H_{q, \Lambda}$ is a selfadjoint operator in this case, $f$ is real. Rewriting the partition function in terms of the $(d+1)$-dimensional contour model introduced in Sect. 3, 
see Eq. (5.2), we have

$$
f=-\frac{1}{\tilde{\beta} M} \lim _{\Lambda \mathbb{Z}^{d}} \frac{1}{|\Lambda|} \log Z_{q}\left(\mathbb{T}_{\Lambda}\right) \equiv-\frac{1}{\tilde{\beta}} \lim _{V \nearrow \mathbb{T}} \frac{1}{|V|} \log Z_{q}(V)
$$

iii) By contrast, the meta-stable free energies $f_{q}$ defined in (5 13) do in general depend on $q$, even if $\lambda$ is real It is worth noting, however, that the meta-stable free energies are real-valued in this case. To see this, we first observe that by the symmetry property $(339), Z_{q}(V)$ is real for all $V$, implying that $K_{q}(Y)^{*}=K_{q}\left(Y^{*}\right)$ Using the definitions $(58)$ through $(5.10)$, one then establishes by induction that $Z_{q}^{\prime}(V)$ is real while $K_{q}^{\prime}(Y)^{*}=K_{q}^{\prime}\left(Y^{*}\right)$. Given this symmetry for $K_{q}^{\prime}(Y)$, the reality of $f_{q}$ now easily follows from the cluster expansion of $\log Z_{q}^{\prime}(V)$.

\section{Expectation Values of Local Variables}

To distinguish between different phases we have to evaluate expectation values of local observables. Whenever we have a local bounded observable $\Psi$, represented by

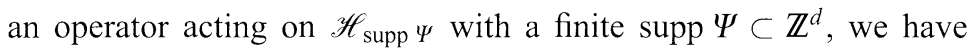

$$
\langle\Psi\rangle_{q, \Lambda}=\frac{\operatorname{Tr}_{\mathscr{H}_{A}}\left(\Psi e^{-\beta H_{q, 1}}\right)}{\operatorname{Tr}_{\mathscr{H}_{1}}\left(e^{-\beta H_{q, 1}}\right)}=\frac{\operatorname{Tr}_{\mathscr{H}_{\Lambda}}\left(\Psi T^{M}\right)}{\operatorname{Tr}_{\mathscr{H}_{, 1}}\left(T^{M}\right)}=\frac{Z_{q, \Lambda}^{\Psi}}{Z_{q, \Lambda}} .
$$

Retracing the steps leading to the contour representation (3.36) of $Z_{q, 1}$, we can get in a straightforward manner a similar expression for $Z_{q, \Lambda}^{\Psi}$ Namely, introducing

$$
w_{\Psi}\left(\Sigma_{1}, \quad, \Sigma_{M}\right)=\operatorname{Tr}_{\mathscr{H}_{\Lambda}}\left(\Psi \prod_{t=1}^{M} K\left(\Sigma_{t}\right)\right)
$$

with $K(\Sigma)$ given as before by (323), we have

$$
Z_{q, \Lambda}^{\Psi}=\sum_{\Sigma_{1}, \Sigma_{M}} w_{\Psi}\left(\Sigma_{1}, \ldots, \Sigma_{M}\right)
$$

Localizing the observable $\Psi$, by definition, in the first time slice, we define the $d+1$ dimensional support of $\Psi$ as

$$
\mathscr{S}(\Psi):=\bigcup_{x \in \operatorname{supp} \Psi} C(x, 1) .
$$

Assuming without loss of generality that supp $\Psi$ and hence $\mathscr{S}(\Psi)$ is a connected set, we introduce the contours corresponding to a configuration $\sigma_{\mathbb{L}_{1}}$ in the same way as before, with the only difference that the supports of these contours are now the connected components of $D \cup \mathscr{S}(\Psi)$, where, as in Sect. 3,D is the set of excited cubes in $\mathbb{T}_{A}$ By this definition, one of the contours corresponding to $\sigma_{\mathbb{L}_{1}}$ will contain the set $\mathscr{S}(\Psi)$ as part of its support We denote this contour by $Y_{\Psi}$ Continuing as before, we obtain the representation

$$
Z_{q, A}^{\Psi}=\sum_{\substack{\left\{Y_{\psi}, Y_{1}, Y_{h}\right\} \\ \operatorname{supp} Y_{\Psi} \supset \mathscr{\mathcal { S }}(\Psi)}} \rho_{\Psi}\left(Y_{\Psi}\right) \prod_{i} \rho\left(Y_{l}\right) \prod_{m} e^{-\tilde{\beta} e_{m}\left|W_{m}\right|},
$$


where

$$
\rho_{\Psi}\left(Y_{\Psi}\right)=\sum_{\sigma_{D} \rightarrow Y_{\Psi}} \omega_{\Psi}\left(\sigma_{D}, \alpha_{D}\right),
$$

with the reduced weight $\omega_{\Psi}$ given by a formula of the type (3.31).

More generally, we introduce, for a volume $V$ which contains the $d+1$ dimensional support $\mathscr{S}(\Psi)$ of $\Psi$, the diluted partition function

$$
Z_{q}^{\Psi}(V)=\sum_{\substack{\left\{Y_{\Psi}, Y_{1},, Y_{k}\right\} \\ \operatorname{supp} Y_{\Psi} \supset S(\Psi)}} \rho \Psi\left(Y_{\Psi}\right) \prod_{i} \rho\left(Y_{i}\right) \prod_{m} e^{-\tilde{\beta} e_{m}\left|W_{m}\right|}
$$

where the sum runs over all sets of mutually compatible, matching contours in $V$ for which all external contours are $q$-contours. For $V=\mathbb{T}_{\Lambda}, Z_{q}^{\Psi}(V)=Z_{q, \Lambda}^{\Psi}$, which implies that

$$
\langle\Psi\rangle_{q, \Lambda}=\frac{Z_{q}^{\Psi}\left(\mathbb{T}_{\Lambda}\right)}{Z_{q}\left(\mathbb{T}_{\Lambda}\right)}
$$

For every term contributing to (6.7) we consider the collection $\mathscr{Y}_{\Psi}$ consisting of the contour $Y_{\Psi}$ as well as all contours $Y$ among $\left\{Y_{1}, \ldots, Y_{k}\right\}$ encircling it (Int $Y \supset$ $\mathscr{S}(\Psi)$ ) and define

$$
\rho\left(\mathscr{Y}_{\Psi}\right)=\rho_{\Psi}\left(Y_{\Psi}\right) \prod_{\substack{Y \in \mathscr{Y}_{\Psi} \\ Y \neq Y_{\Psi}}} \rho(Y)
$$

Denoting further supp $\mathscr{Y}_{\Psi}=\bigcup_{Y \in \mathscr{Y}_{\Psi}} \operatorname{supp} Y$, Int $\mathscr{Y}_{\Psi}$ the union of all finite components of $\mathbb{T} \backslash \operatorname{supp} \mathscr{Y}_{\Psi}, \operatorname{Int}_{m} \mathscr{Y}_{\Psi}$ the union of all components of Int $\mathscr{Y}_{\Psi}$ that are labeled by $m$, and Ext $\mathscr{Y}_{\Psi}=\bigcap_{Y \in \mathscr{Y}_{\Psi}}$ Ext $Y$, we introduce, in addition to the weights $K_{q}(Y)$ defined in the preceding section for an arbitrary $q$-contour $Y$, also the weight

$$
K_{q, \Psi}\left(\mathscr{Y}_{\Psi}\right):=\rho_{\Psi}\left(\mathscr{Y}_{\Psi}\right) e^{\tilde{\beta} e_{q}\left|\operatorname{supp} \mathscr{Y}_{\Psi}\right|} \prod_{m=1}^{r} \frac{Z_{m}\left(\operatorname{Int}_{m} \mathscr{Y}_{\Psi}\right)}{Z_{q}\left(\operatorname{Int}_{m} \mathscr{Y}_{\Psi}\right)}
$$

attributed to the collection $\mathscr{Y}_{\Psi}$. As a result we get the representation

$$
Z_{q}^{\Psi}(V)=e^{-\tilde{\beta} e_{q}|V|} \sum_{\mathscr{Y}_{\Psi}, Y_{1},, Y_{n}} K_{q, \Psi}\left(\mathscr{Y}_{\Psi}\right) \prod_{l=1}^{n} K_{q}\left(Y_{l}\right)
$$

Here the sum goes over set of all collections $\mathscr{Y}_{\Psi}$ and all sets $\left\{Y_{1}, \ldots, Y_{n}\right\}$ of nonoverlapping $q$ contours in $V$, such that for all contours $Y_{i}, i=1, \ldots, n$, the set $V\left(Y_{i}\right)$ does not intersect the set supp $\mathscr{Y}_{\Psi}$.

Assuming for the moment that the weights $K_{q, \Psi}\left(\mathscr{Y}_{\Psi}\right)$ and $K_{q}(Y)$ decay sufficiently fast with the size of $\mathscr{Y}_{\Psi}$ and $Y$, respectively, we now use the standard Mayer cluster expansion for polymer systems to get

$$
\frac{Z_{q}^{\Psi}(V)}{Z_{q}(V)}=\sum_{\mathscr{Y}_{\Psi}} K_{q, \Psi}\left(\mathscr{Y}_{\Psi}\right) \sum_{n=0}^{\infty} \frac{1}{n !} \sum_{\left\{Y_{1}, Y_{n}\right\}}\left[\prod_{k=1}^{n} K_{q}\left(Y_{k}\right)\right] \phi_{c}\left(\mathscr{Y}_{\Psi}, Y_{1}, \ldots, Y_{n}\right) .
$$

Here $\phi_{c}\left(\mathscr{Y}_{\Psi}, Y_{1}, \ldots, Y_{n}\right)$ is a combinatoric factor defined in terms of the connectivity properties of the graph $G\left(\mathscr{Y}_{0}, Y_{1}, \ldots, Y_{n}\right)$, introduced as the graph on the vertex set $\{0,1, \ldots, n\}$ which has an edge between two vertices $i \geqq 1$ and $j \geqq 1, i \neq j$, whenever supp $Y_{i} \cap \operatorname{supp} Y_{j} \neq \emptyset$, and an edge between the vertex 0 and a vertex $i \neq 0$ whenever $V\left(Y_{i}\right) \cap \operatorname{supp} \mathscr{Y}_{\Psi} \neq \emptyset$ (see for example [Sei82] or [Dob94] for a new 
simple and very lucid proof). The combinatoric factor $\phi_{c}\left(\mathscr{Y}_{\Psi}, Y_{1}, ., Y_{n}\right)$ is zero if $G\left(Y_{\Psi}, Y_{1}, \ldots, Y_{n}\right)$ has more than one component.

To prove the convergence of (6.12) one has to show that the weights $K_{q, \Psi}$ and $K_{q}$ decay sufficiently fast with the size of $\mathscr{Y}_{\Psi}$ and $Y$, respectively. To this end it is useful to introduce truncated models. For a contour $Y$ with $V(Y) \cap \mathscr{S}(\Psi)=\emptyset$, we define $K_{q}^{\prime}(Y)$ as before, see (5.9), while for the collection $\mathscr{Y}_{\Psi}$ we define

$$
K_{q, \Psi}^{\prime}\left(\mathscr{Y}_{\Psi}\right)=\rho \Psi\left(\mathscr{Y}_{\Psi}\right) e^{\tilde{\beta} e_{q}\left|\operatorname{supp} \mathscr{Y}_{\Psi}\right|} \prod_{m=1}^{\prime} \frac{Z_{m}\left(\operatorname{Int}_{m} \mathscr{Y}_{\Psi}\right)}{Z_{q}^{\prime}\left(\operatorname{Int}_{m} \mathscr{Y}_{\Psi}\right)} \prod_{Y \in \mathscr{Y}_{\Psi}} \chi_{q}^{\prime}(Y),
$$

with $\chi_{q}^{\prime}(Y)$ as in (5 10) Given this definition, we introduce

$$
Z_{q}^{\prime \Psi}(V)=e^{-\tilde{\beta} e_{q}|V|} \sum_{\mathscr{Y}_{\Psi}} K_{q, \Psi}^{\prime}\left(\mathscr{Y}_{\Psi}\right) \sum_{\left\{Y_{1}, Y_{n}\right\}} \prod_{k=1}^{n} K_{q}^{\prime}\left(Y_{k}\right)
$$

and

$$
\langle\Psi\rangle_{q, V}^{\prime}=\frac{Z_{q}^{\prime \Psi}(V)}{Z_{q}^{\prime}(V)}
$$

which can again be expanded as

$$
\langle\Psi\rangle_{q, V}^{\prime}=\sum_{\mathscr{Y}_{\Psi}} K_{q, \Psi}^{\prime}\left(\mathscr{Y}_{\Psi}\right) \sum_{n=0}^{\infty} \frac{1}{n !} \sum_{\left\{Y_{1},, Y_{n}\right\}}\left[\prod_{k=1}^{n} K_{q}^{\prime}\left(Y_{k}\right)\right] \phi_{c}\left(\mathscr{Y}_{\Psi}, Y_{1}, \quad, Y_{n}\right)
$$

The following lemma gives the absolute convergence of the expansion (6.16), which in turn will yield Theorem 2.1 ii), iii) and iv).

Lemma 6.1. Let $\varepsilon, \varepsilon_{0}$ and $\bar{\alpha}$ be as in Lemma 51 , and assume that $\varepsilon<\varepsilon_{0}$ and $\bar{\alpha} \geqq 1$ Then

i) For every collection $\mathscr{Y}_{\Psi}$ one has

$$
\left|K_{q, \Psi}^{\prime}\left(\mathscr{Y}_{\Psi}\right)\right| \leqq\|\Psi\| e^{(\gamma+2)|\operatorname{supp} \Psi|} \varepsilon_{\varepsilon}^{|\operatorname{supp} \mathscr{\mathscr { H }} \backslash \backslash \mathscr{P}(\Psi)|} .
$$

ii) If

$$
a_{q} \max _{Y \in \mathscr{P} \psi_{\psi}} \delta(Y) \leqq \bar{\alpha}
$$

then $K_{q, \Psi}^{\prime}\left(\mathscr{Y}_{\Psi}\right)=K_{q, \Psi}\left(\mathscr{Y}_{\Psi}\right)$

iii) The cluster expansion (6.16) is absolutely convergent, and

$$
\begin{aligned}
\left|\langle\Psi\rangle_{q, V}^{\prime}\right| & \leqq \sum_{\mathscr{Y}_{\Psi}}\left|K_{q, \Psi}^{\prime}\left(\mathscr{Y}_{\Psi}\right)\right| \sum_{n=0}^{\infty} \frac{1}{n !} \sum_{\left\{Y_{1}, Y_{n}\right\}}\left[\prod_{k=1}^{n}\left|K_{q}^{\prime}\left(Y_{k}\right)\right|\right]\left|\phi_{c}\left(\mathscr{Y}_{\Psi}, Y_{1}, \ldots, Y_{n}\right)\right| \\
& \leqq\|\Psi\| e^{(\alpha+2+O(\varepsilon))|\operatorname{supp} \Psi|}
\end{aligned}
$$

iv) If $a_{q} \delta\left(\mathbb{T}_{\Lambda}\right) \leqq \bar{\alpha}$, then $\langle\Psi\rangle_{q, \Lambda}=\langle\Psi\rangle_{q, \Lambda}^{\prime}$.

v) For an arbitrary volume $V \subset \mathbb{T}$,

$$
\left|Z_{q}^{\Psi}(V)\right| \leqq\|\Psi\| e^{(\gamma+1)|\operatorname{supp} \Psi|} e^{-\tilde{\beta} f_{0}|V|+O(\varepsilon)|\hat{\alpha} V|} .
$$

vi) There is a constant $K=K(d)>0$ such that for $V=\mathbb{T}_{\Lambda}$,

$$
\left.\left|Z_{q}^{\Psi}(V)\right| \leqq\|\Psi\| e^{(\gamma+1)|\operatorname{supp} \Psi|} e^{-\tilde{\beta} f_{0}|V|+O(\varepsilon)|\hat{\partial} V|} \max e^{-\frac{a_{q}}{4}|V|}, e^{-K \gamma|\partial V|}\right)
$$


Proof. Observing that $\delta(W) \leqq \max _{Y \in \mathscr{Y}_{\Psi}} \delta(Y)$ for all components $W$ of Int $\mathscr{Y}_{\Psi}$, the statement ii) of Lemma 6.1 immediately follows from Lemma 5.1.

In order to prove i), we first note that $\rho_{\Psi}\left(Y_{\Psi}\right) \leqq\|\Psi\| \tilde{\rho}\left(Y_{\Psi}\right)$, where $\tilde{\rho}\left(Y_{\Psi}\right)$ satisfies an analog of the bound (4.4) (however, $Y_{\Psi}$ is not necessarily excited on $\mathscr{S}(\Psi))$, namely

$$
\left|\tilde{\rho}\left(Y_{\Psi}\right)\right| \leqq e^{-\tilde{\beta} e_{0}\left|\operatorname{supp} Y_{\Psi}\right|} e^{-\gamma\left|\operatorname{supp} Y_{\Psi} \backslash \mathscr{S}(\Psi)\right|}\left(1+e^{-\gamma}\right)^{|\mathscr{S}(\Psi)|}
$$

Hence

$$
\rho_{\Psi}\left(\mathscr{Y}_{\Psi}\right) e^{\tilde{\beta} e_{q}\left|\operatorname{supp} \mathscr{Y}_{\Psi}\right|} \leqq\|\Psi\| e^{-\tilde{\beta}\left(e_{0}-e_{q}\right)\left|\operatorname{supp} \mathscr{Y}_{\Psi}\right|} e^{-\gamma\left|\operatorname{supp} \mathscr{Y}_{\Psi} \backslash \mathscr{S}(\Psi)\right|}\left(1+e^{-\gamma}\right)^{|\mathscr{S}(\Psi)|}
$$

Using Lemma 5.1 we get

$$
\prod_{m=1}^{r}\left|\frac{Z_{m}\left(\operatorname{Int} \mathscr{Y}_{\Psi}\right)}{Z_{q}^{\prime}\left(\operatorname{Int} \mathscr{Y}_{\Psi}\right)}\right| \leqq e^{a_{q}\left|\operatorname{Int} \mathscr{Y}_{\Psi}\right|+O(\varepsilon)\left|\partial \operatorname{Int} \mathscr{Y}_{\Psi}\right|}
$$

Combined with the bound $\tilde{\beta}\left(e_{q}-e_{0}\right) \leqq a_{q}+O(\varepsilon)$ we get

$$
\left|K_{q, \Psi}^{\prime}\left(\mathscr{Y}_{\Psi}\right)\right| \leqq\|\Psi\| e^{a_{q}\left|\operatorname{supp} \mathscr{Y}_{\Psi} \cup \operatorname{Int} \mathscr{Y}_{\Psi}\right|+O(\varepsilon)\left|\partial \operatorname{Int} \mathscr{Y}_{\Psi}\right|} e^{-\gamma\left|\operatorname{supp} \mathscr{Y}_{\Psi} \backslash \mathscr{S}(\Psi)\right|} e^{O(\varepsilon)\left|\operatorname{supp} \mathscr{Y}_{\Psi}\right|} .
$$

Observing that $\prod_{Y \in \mathscr{Y}_{\Psi}} \chi_{q}(Y) \neq 0$ implies that $a_{q}|V(Y)| \leqq(\alpha+1+O(\varepsilon))|Y|$ for each $Y \in \mathscr{Y}_{\Psi}$, and noting that $|\mathscr{S}(\Psi)|=|\operatorname{supp} \Psi|$, we finally get

$$
\left|K_{q, \Psi}^{\prime}\left(\mathscr{Y}_{\Psi}\right)\right| \leqq\|\Psi\| e^{-(\gamma-\alpha-1-O(\varepsilon))\left|\operatorname{supp} \mathscr{Y}_{\Psi} \backslash \mathscr{S}(\Psi)\right|} e^{(\alpha+1+O(\varepsilon))|\operatorname{supp} \Psi|} .
$$

Statement iii) follows from i) and Lemma 5.1, i), while statement iv) follows from ii) and Lemma 5.1, ii) and iii).

To get the statement v) and vi), we first write $Z_{q}^{\Psi}(V)$ as

$$
Z_{q}^{\Psi}(V)=\sum_{\mathscr{Y}_{\Psi}} \rho_{\Psi}\left(\mathscr{Y}_{\Psi}\right) Z_{q}\left(\operatorname{Ext} \mathscr{Y}_{\Psi}\right) \prod_{m} Z_{m}\left(\operatorname{Int} \mathscr{Y}_{\Psi}\right) .
$$

Using now (6.23) and the bound (5.18) in its strengthened form (A.43), we get

$$
\begin{gathered}
\left|Z_{q}^{\Psi}(V)\right| \leqq\|\Psi\| e^{\gamma|\mathscr{S}(\Psi)|} e^{-\tilde{\beta} f_{0}|V|} e^{O(\varepsilon)|\partial V|} \sum_{\mathscr{Y}_{\Psi}} e^{-\gamma\left|\operatorname{supp} \mathscr{Y}_{\Psi}\right|} e^{O(\varepsilon) \mid \text { supp } \mathscr{Y}_{\Psi} \mid} \\
\times \max _{U \subset \operatorname{Ext} \mathscr{Y}_{\Psi}}\left(e^{-\frac{a_{q}}{4}\left|\operatorname{Ext} \mathscr{O}_{\Psi} \backslash U\right|} e^{-K(d) \gamma|\partial U|}\right) .
\end{gathered}
$$

Extracting a factor

$$
\begin{aligned}
& \max _{\mathscr{Y}_{\Psi}} e^{-\frac{\gamma}{2}\left|\operatorname{supp} \mathscr{Y}_{\Psi}\right|} \max _{U \subset \operatorname{Ext} \mathscr{Y}_{\Psi}}\left(e^{-\frac{a_{q}}{4}\left|\operatorname{Ext} \mathscr{Y}_{\Psi} \backslash U\right|} e^{-K(d) \gamma|\partial U|}\right) \\
& \leqq \max _{\mathscr{Y}_{\Psi}} e^{-K(d) \gamma\left|\partial V\left(\mathscr{Y}_{\Psi}\right)\right|} \max _{U \subset V \backslash V\left(\mathscr{Y}_{\Psi}\right)}\left(e^{-\frac{a_{q}}{4}\left|\left(V \backslash V\left(\mathscr{Y}_{\Psi}\right)\right) \backslash U\right|} e^{-K(d) \gamma|\partial U|}\right) \\
& \leqq \max _{\tilde{U} \subset V}\left(e^{-\frac{a_{q}}{4}|V \backslash \tilde{U}|} e^{-K(d) \gamma|\partial \tilde{U}|}\right)
\end{aligned}
$$

where $V\left(\mathscr{Y}_{\Psi}\right)=V \backslash \operatorname{Ext} \mathscr{Y}_{\Psi}$, and bounding

$$
\sum_{\mathscr{Y}_{\Psi}} e^{-\frac{\gamma}{2}\left|\operatorname{supp} \mathscr{Y}_{\Psi}\right|} \leqq\left(1+O\left(e^{-\frac{\gamma}{2}}\right)\right)^{|\operatorname{supp} \Psi|},
$$


we get

$$
\left|Z_{q}^{\Psi}(V)\right| \leqq\|\Psi\| e^{(i+1)|\operatorname{supp} \Psi|} e^{-\tilde{\beta} f_{0}|V|} e^{O(\xi)|\hat{\partial} V|} \max _{\tilde{U} \subset V}\left(e^{-\frac{a_{q}}{4}|V \backslash \tilde{U}|} e^{-K(d)_{i}|\tilde{C}|}\right)
$$

Bounding the last factor by one, we get statement v), continuing as in the proof of Lemma A 3 in the appendix, we get statement vi)

\section{Proof of Theorems 2.1 and 2.2}

Proof of Theorem 21 In order to prove the theorem, we will use Lemmas 5.1 and Lemma 61 , with $\alpha=\gamma / 2$. We therefore need that $\gamma>4-2 \log \varepsilon_{0}$, which in turn requires $\tilde{\beta}$ and $\gamma_{Q}$ are sufficiently large, see (4.5). Choosing appropriate $\beta_{0}$ and $\gamma_{Q}$, we take $\tilde{\beta} \in\left(\frac{1}{2} \beta_{0}, \beta_{0}\right]$ and choose $\lambda_{0}$ according to (2.23). Then (4.3) and (4 21) are satisfied once $|\lambda| \leqq \lambda_{0}$. For every $\beta \geqq \beta_{0}$ we now choose $M$ such that $\tilde{\beta}=\frac{\beta}{M} \in\left(\frac{1}{2} \beta_{0}, \beta_{0}\right]$ Whenever $a_{q}=0$ we can then use the claims ii) from Lemma 51 and ii) from Lemma 6.1 to conclude that $K_{q}(Y)=K_{q}^{\prime}(Y)$ for all $Y$ and $K_{q, \psi}^{\prime}\left(\mathscr{Y}_{\Psi}\right)=$ $K_{q, \Psi}\left(\mathscr{Y}_{\Psi}\right)$ for all $\mathscr{Y}_{\Psi}$. As a result the bounds (5 5), (5.17), and (6.17) are valid for $K_{q}(Y)$ and $K_{q, \psi}\left(\mathscr{Y}_{\Psi}\right)$. This allows us to use cluster expansions for evaluation of $Z_{q}(V)$ as well as $Z_{q}^{\Psi}(V)$.

Hence (2.25) follows directly from (5.2), (5.13) and Lemma 5.1 iii). Similarly, we get (2.26) (with an explicit formula for $\langle\Psi\rangle_{q}$ ) from (6.12) Finally, given the absolute convergence of the cluster expansion for the expectation values of local observables and the exponential decay of the contour activities, the bound (2.27) is standard

To evaluate the expectation value of the projection operator (2 28), we apply the expansion (6 10) for the particular observable $\Psi=P_{U(x)}^{(m)} \equiv\left|g_{U(r)}^{(m)}\right\rangle\left\langle g_{U(x)}^{(m)}\right|$ Using the factor $\varepsilon^{\left|\operatorname{supp} \mathscr{M}_{\Psi} \backslash \mathscr{S}(\Psi)\right|}$ in (6.17), one can show that the sum of all terms with $\operatorname{supp} \mathscr{Y}_{\Psi} \neq \mathscr{S}(\Psi)$ is of the order const $\varepsilon$, a term that can be made small by taking $\varepsilon$ small We are thus left with contributions coming from the term $K_{q, \Psi}\left(\mathscr{Y}_{\Psi}\right)$ with

$$
\operatorname{supp} \mathscr{Y}_{\Psi}=\mathscr{S}(\Psi) \equiv \bigcup_{v \in U(x)} C(y, 1)
$$

This means that necessarily $\mathscr{Y}_{\Psi}=\left\{Y_{\Psi}\right\}$ with supp $Y_{\Psi}=\mathscr{S}(\Psi)$ and $\alpha(\partial \mathscr{S}(\Psi))=q$ The only configurations $\sigma$ yielding this $Y_{\Psi}$ are those for which $\sigma=\left\{\sigma_{y, t}\right\}$ agrees with $g^{(q)}$, except possibly for the point $(y, t)=(x, 1)$. For the activity

$$
K_{q, \Psi}\left(\mathscr{Y}_{\Psi}\right)=\rho_{\Psi}\left(\left\{Y_{\Psi}\right\}\right) e^{\tilde{\beta} e_{q}|U(x)|},
$$

this gives a contribution $O(\varepsilon)$ if $\sigma_{x, 1} \neq g_{x}^{(q)}$, and a contribution

$$
\begin{aligned}
& e^{\tilde{\beta} e_{q}|\Lambda| M} \operatorname{Tr}_{\mathscr{H}_{1}}\left(\Psi\left|g_{\Lambda}^{(q)}\right\rangle\left\langle g_{\Lambda}^{(q)}\right| \prod_{t=1}^{M} e^{-\tilde{\beta} \sum_{v \in 1}} \Phi_{y}\left(g_{U(y)}^{(q)}\right)\right) \\
& =\operatorname{Tr}_{\mathscr{H}_{1}}\left(\Psi\left|g_{\Lambda}^{(q)}\right\rangle\left\langle g_{\Lambda}^{(q)}\right|\right)=\delta_{m, q},
\end{aligned}
$$


if $\sigma_{x, 1}=g_{x}^{(q)}$. Putting everything together, we obtain the bound

$$
\left\langle P_{U(x)}^{(m)}\right\rangle_{q}=\delta_{m, q}+O(\varepsilon)
$$

which proves iv).

Finally, v) is a standard claim in the Pirogov-Sinai theory. Namely, given the bounds (5.5), (5.17), and the fact that $f_{q}$ can be analysed by a convergent cluster expansion, we get $\left|f_{q}-e_{q}\right| \leqq O(\varepsilon)$ and a similar bound for the derivatives of $f_{q}$. Statement $v$ ) then follows from the corresponding assumptions (2.4) and (2.5) on the functions $e_{q}(\mu)$.

Proof of Theorem 2.2. The partition functions

$$
Z_{\text {per }, A(L)}=\operatorname{Tr}_{\mathscr{H}_{A}} e^{-\beta H_{\text {per }, A(L)}}
$$

and

$$
Z_{\mathrm{per}, \Lambda(L)}^{\Psi}=\operatorname{Tr}_{\mathscr{H}_{\Lambda}} \Psi e^{-\beta H_{\mathrm{per}, \Lambda(L)}}
$$

have representations $Z\left(\mathbb{T}_{\Lambda_{\text {per }}(L)}\right)$ and $Z^{\Psi}\left(\mathbb{T}_{\Lambda_{\text {per }}(L)}\right)$ similar to (5.1) and (6.5). The proof of Theorem 2.2 follows from this representation in a standard manner [BI89, BK90]. One only has to notice that contours contributing to these partition functions may be wrapped around the torus $\mathbb{T}_{\Lambda_{\text {per }}(L)}$ in time as well as space directions. Nevertheless, whenever a contour $Y$ satisfies the condition $\delta(Y) \leqq \frac{L}{3}$, one can define Ext $Y$ as the largest component of $\mathbb{T}_{\Lambda_{\text {per }}} \backslash \operatorname{supp} Y$. For every configuration containing only such contours, all external contours have clearly the same external label. Splitting now $Z\left(\mathbb{T}_{\Lambda_{\mathrm{per}}(L)}\right)$ (resp. $\left.Z^{\Psi}\left(\mathbb{T}_{\Lambda_{\text {per }}(L)}\right)\right)$ into contributions containing at least one contour such that $\delta(Y)>\frac{L}{3}$ and those where all contributing contours are such that $\delta(Y) \leqq \frac{L}{3}$, we get

$$
Z\left(\mathbb{T}_{\Lambda_{\mathrm{per}}}\right)=Z^{\mathrm{big}}\left(\mathbb{T}_{\Lambda_{\mathrm{per}}}\right)+\sum_{m=1}^{r} Z_{m}\left(\mathbb{T}_{\Lambda_{\mathrm{per}}}\right)
$$

Here $Z_{m}\left(\mathbb{T}_{\Lambda_{\text {per }}}\right)$ is given as a sum over all configurations containing only those contours for which $\delta(Y) \leqq \frac{L}{3}$ and such that the common external label of external contours is $m$. Taking into account the fact that term $Z^{\text {big }}\left(\mathbb{T}_{\Lambda_{\text {per }}}\right)$ is exponentially suppressed in $L$ (one can use verbatim the proof from [BI89]), we get

$$
\left|Z\left(\mathbb{T}_{\Lambda_{\mathrm{per}}}\right)-\sum_{m=1}^{r} Z_{m}\left(\mathbb{T}_{\Lambda_{\mathrm{per}}}\right)\right| \leqq e^{-\tilde{\beta} f_{0} M(2 L+1)^{d}} e^{- \text {const } \gamma L}
$$

and similarly

$$
\left|Z^{\Psi}\left(\mathbb{T}_{\Lambda_{\mathrm{per}}}\right)-\sum_{m=1}^{r} Z_{m}^{\Psi}\left(\mathbb{T}_{\Lambda_{\mathrm{per}}}\right)\right| \leqq\|\Psi\| e^{(\gamma+1)|\operatorname{supp} \Psi|} e^{-\tilde{\beta} f_{0} M(2 L+1)^{d}} e^{- \text {const } \gamma L}
$$

Moreover, whenever $m$ is stable, $m \in Q(\mu)$, we have $Z_{m}\left(\mathbb{T}_{\Lambda_{\text {per }}}\right)=Z_{m}^{\prime}\left(\mathbb{T}_{\Lambda_{\text {per }}}\right)$ and we can use the cluster expansion of $\log Z_{m}^{\prime}\left(\mathbb{T}_{\Lambda_{\text {per }}}\right)$ and $f_{m}$ to show that

$$
\left|Z_{m}\left(\mathbb{T}_{\Lambda_{\mathrm{per}}}\right)-e^{-\tilde{\beta} f_{m} M(2 L+1)^{d}}\right| \leqq e^{-\tilde{\beta} f_{0} M(2 L+1)^{d}} M(2 L+1)^{d} e^{- \text {const } \gamma L} .
$$


Namely, we just observe that the first terms in which these two cluster expansions differ are of the order $e^{- \text {const } ; L}$ (clusters wrapped around $\mathbb{T}_{\Lambda_{\mathrm{pct}}}$ in spatial directions)

For $m \notin Q(\mu)$, on the other hand, we proceed as in the proof of Lemma A.3 to get

$$
\begin{aligned}
\left|Z_{m}\left(\mathbb{T}_{\Lambda_{\mathrm{pcl}}}\right)\right| \leqq & \exp \left(M(2 L+1)^{d} e^{-\mathrm{const} ; L}\right) e^{-\tilde{\beta} f_{0} M(2 L+1)^{d}} \\
& \times \max \left\{e^{-\frac{u_{q}}{4} M(2 L+1)^{d}}, e^{-K(d)_{;} M(2 L+1)^{d-1}}\right\} .
\end{aligned}
$$

For $m \notin Q(\mu)$ and $L$ sufficiently large, we therefore get

$$
\left|Z_{m}\left(\mathbb{T}_{\Lambda_{\mathrm{pcl}}}\right)\right| \leqq 2 e^{-\tilde{\beta} f_{0} M(2 L+1)^{d}} e^{-K(d) ; M(2 L+1)^{d-1}}
$$

In a similar way, proceeding now as in the proof of Lemma 6.1, we get

$$
\left|Z_{m}^{\Psi}\left(\mathbb{T}_{\Lambda_{\mathrm{pcr}}}\right)\right| \leqq 2\|\Psi\| e^{(;+1)|\operatorname{supp} \Psi|} e^{-\tilde{\beta} f_{0} M(2 L+1)^{d}} e^{-K(d)_{i} M(2 L+1)^{d-1}},
$$

provided $m \notin Q(\mu)$ and $L$ is sufficiently large.

Combining the bounds (78), (7.10) and (7 12), and using the fact that $f_{m}=$ $\operatorname{Re} f_{m}$ if the coupling constant $\lambda$ is real, which in turn implies $f_{m}=f_{0}$ if $m \in Q(\mu)$, we get

$$
\left|Z\left(\mathbb{T}_{\Lambda_{\mathrm{pcl}}}\right)-\right| Q(\mu)\left|e^{-\tilde{\beta} f_{0} M(2 L+1)^{d}}\right| \leqq e^{-\tilde{\beta} f_{0} M(2 L+1)^{d}} O\left(e^{- \text {const } \tau_{i} L}\right),
$$

provided $L$ is sufficiently large.

Introducing now the truncated expectation values

$$
\langle\Psi\rangle_{q, \mathbb{T}_{\mathrm{p}_{\mathrm{pcl}}}}^{\prime}=\frac{Z_{q}^{\prime \Psi}\left(\mathbb{T}_{\Lambda_{\mathrm{pct}}}\right)}{Z_{q}^{\prime}\left(\mathbb{T}_{\Lambda_{\mathrm{pcr}}}\right)}
$$

(cf (5 6) and (6 14)), we get

$$
\begin{aligned}
& \left|Z^{\Psi}\left(\mathbb{T}_{\Lambda_{\mathrm{pel}}}\right)-\sum_{m \in Q(\mu)} e^{-\tilde{\beta} \operatorname{Re} f_{m} M(2 L+1)^{d}}\langle\Psi\rangle_{m, \mathbb{T}_{\mathrm{p}_{\mathrm{pel}}}^{\prime}}\right| \\
& \leqq \sum_{m \in Q(\mu)}\left|\langle\Psi\rangle_{m, \mathbb{T}_{i_{\mathrm{pc1}}}^{\prime}}^{\prime}\left(Z_{m}^{\prime}\left(\mathbb{T}_{\Lambda_{\mathrm{pc1}}}\right)-e^{-\tilde{\beta} \operatorname{Re} f_{m} M(2 L+1)^{d}}\right)\right| \\
& +\sum_{m \notin Q(\mu)}\left|Z_{m}^{\Psi}\left(\mathbb{T}_{\Lambda_{\mathrm{pel}}}\right)\right|+\|\Psi\| e^{(i+1)|\operatorname{supp} \Psi|} e^{-\tilde{\beta} f_{0} M(2 L+1)^{d}} e^{- \text {const }{ }^{\prime} L} .
\end{aligned}
$$

Next, we observe that $\langle\Psi\rangle_{m, \mathbb{T}_{1 \mathrm{pcr}}}^{\prime}$ can be bounded in the same way as $\langle\Psi\rangle_{m, \mathbb{T}_{1}}^{\prime}$, namely

$$
\left|\langle\Psi\rangle_{m, \mathbb{T}_{A_{\mathrm{pcl}}}}^{\prime}\right| \leqq\|\Psi\| e^{(\gamma+2+O(\varepsilon))|\operatorname{supp} \Psi|} \leqq\|\Psi\| e^{(i+1)|\operatorname{supp} \Psi|}
$$

Inserting the bounds (7.10), (7 17) and (7 13) into (7 16), and dividing both sides of the resulting bound by $|Q(\mu)| e^{-\tilde{\beta} f_{0} M(2 L+1)^{d}}$, we get

$$
\left|\langle\Psi\rangle_{\mathrm{pcl}, A(L)}-\sum_{m \in Q(\mu)}\langle\Psi\rangle_{m, \mathbb{T}_{1 \mathrm{pcl}}}\right| \leqq e^{- \text {const } ; L}\|\Psi\| e^{(;+1) \mid \text { supp } \Psi \mid},
$$

provided $L$ is sufficiently large In the limit $L \rightarrow \infty$, this yields the claim of Theorem 22. 


\section{Appendix. Pirogov-Sinai Theory in Thin Slabs - Proof of Lemma 5.1}

In this appendix, we prove Lemma 5.1. Actually, it is a direct consequence of Lemma A.1 below. In order to state the lemma, we recall the definition of $f_{q}^{(n)}$ as the free energy of an auxiliary contour model with activities

$$
K^{(n)}(Y)= \begin{cases}K^{\prime}(Y) & \text { if } \delta(Y) \leqq n \\ 0 & \text { otherwise }\end{cases}
$$

and define

$$
\begin{gathered}
f_{0}^{(n)}=\min _{q} \operatorname{Re} f_{q}^{(n)}, \\
a_{q}^{(n)}=\tilde{\beta}\left(\operatorname{Re} f_{q}^{(n)}-f_{0}^{(n)}\right) .
\end{gathered}
$$

Observing that $f_{0}=\lim _{n \rightarrow \infty} f_{0}^{(n)}$ and $a_{q}=\lim _{n \rightarrow \infty} a_{q}^{(n)}$, Lemma 5.1 follows directly from the following.

Lemma A.1. Assume that $\rho(\cdot)$ obeys the conditions (4.4) and (4.22) and let

$$
\varepsilon=e^{-\gamma+\alpha+2} \text { and } \bar{\alpha}=\alpha-2 .
$$

Then there is a constant $\varepsilon_{0}$, depending only on $d$ and $r$, such that the following statements are true once ( $\gamma$ is such that) $\varepsilon<\varepsilon_{0}$ and $\alpha \geqq 3$. For all $n \geqq 0$ and $Y$ and $V$ such that $\delta(Y) \leqq n, \delta(V) \leqq n$, one has:

i) $\left|K_{q}^{\prime}(Y)\right| \leqq \varepsilon^{|Y|}$,

ii) $\left|\frac{\partial}{\partial \mu_{t}} K_{q}^{\prime}(Y)\right| \leqq\left(3 r \tilde{\beta} C_{0}+2\right)|V(Y)| \varepsilon^{|Y|}$,

iii) If $a_{q}^{(n)} \delta(Y) \leqq \bar{\alpha}$ then $\chi_{q}^{\prime}(Y)=1$,

iv) If $a_{q}^{(n)} \delta(Y) \leqq \bar{\alpha}$ then $K_{q}^{\prime}(Y)=K_{q}(Y)$,

v) If $a_{q}^{(n)} \delta(V) \leqq \bar{\alpha}$ then $Z_{q}^{\prime}(V)=Z_{q}(V)$,

vi) $\left|Z_{q}(V)\right| \leqq e^{-\tilde{\beta} f_{0}^{(n)}|V|} e^{O(\varepsilon)|\partial V|}$,

vii) $\left|\frac{\partial}{\partial \mu_{l}} Z_{q}(V)\right| \leqq\left(2 \tilde{\beta} C_{0}+1\right)|V| e^{-\tilde{\beta} f_{0}^{(n)}|V|} e^{O(\varepsilon)|\partial V|}$.

Proof. We proceed by induction on $n$.

I. The case $n=0$. There are no contours with $\delta(Y)=0$. This makes i)-iv) trivial statements and implies that $f_{q}^{(0)}=e_{q}$. On the other hand, $\delta(V)=0$ implies $|V|=0$ and $Z_{q}(V)=Z_{q}^{\prime}(V)=1$, which makes v)-vii) trivial statements.

\section{Induction step $n-1 \rightarrow n$.}

Proof of i) for $\delta(Y)=n$. Clearly, $\delta(\operatorname{Int} Y)<n$, and all contours $\tilde{Y}$ contributing to $Z_{q}^{\prime}\left(\operatorname{Int}_{m} Y\right)$ obey the condition $\delta(\tilde{Y})<n$. This implies that $\left|K_{q}^{\prime}(\tilde{Y})\right| \leqq \varepsilon^{|\tilde{Y}|}$ by the inductive assumption i). As a consequence, the logarithm of $Z_{q}^{\prime}\left(\operatorname{Int}_{m} Y\right)$ can be analyzed by a convergent expansion, and

$$
\left|\log Z_{q}^{\prime}\left(\operatorname{Int}_{m} Y\right)+\tilde{\beta} f_{q}^{(n-1)}\right| \operatorname{Int}_{m} Y|| \leqq O(\varepsilon)\left|\partial \operatorname{Int}_{m} Y\right| .
$$


Combining (A 5) with the induction assumption vi), we get

$$
\prod_{m}\left|\frac{Z_{m}\left(\operatorname{Int}_{m} Y\right)}{Z_{q}^{\prime}\left(\operatorname{Int}_{m} Y\right)}\right| \leqq e^{a_{q}^{(n-1)}|\operatorname{Int} Y|} e^{O(\varepsilon) \sum_{m}\left|\hat{\partial} \operatorname{Int}_{m} Y\right|} \leqq e^{a_{q}^{(n-1)}|\operatorname{Int} Y|} e^{O(\varepsilon)|Y|}
$$

Observing that

$$
\left|\tilde{\beta} e_{m}-\tilde{\beta} f_{m}^{(n-1)}\right| \leqq O(\varepsilon)
$$

which implies the bound

$$
\left|\tilde{\beta}\left(e_{q}-e_{0}\right)-a_{q}^{(n-1)}\right| \leqq O(\varepsilon)
$$

we use the bound (4.4) to evaluate

$$
\left|\rho(Y) e^{\tilde{\beta} e_{q}|Y|}\right| \leqq e^{-\hat{\eta}|Y|} e^{\tilde{\beta}\left(e_{q}-e_{0}\right)|Y|} \leqq e^{-(i-O(\varepsilon))|Y|} e^{a_{q}^{(n-1)}|Y|}
$$

Applying now the bounds (A.9) and (A.6) to the definition (5.9) and using the equation $|V(Y)|=|\operatorname{Int} Y|+|Y|$, we obtain

$$
\left|K_{q}^{\prime}(Y)\right| \leqq \chi_{q}^{\prime}(Y) e^{a_{q}^{(n-1)}|V(Y)|} e^{-(\gamma-O(\varepsilon))|Y|}
$$

Without loss of generality, we may assume that $\chi_{q}^{\prime}(Y)>0$ (otherwise $K_{q}^{\prime}(Y)=0$ and the statement $i$ ) is trivial) Let us notice that

$$
|V(Y)| \leqq \delta(Y)|Y|
$$

Indeed, considering a disjoint union $T$ of "rows" consisting of elementary cubes $C(x, t)$ with fixed cordinates $x_{2}, \ldots, x_{d}$ and $t$, one notices that the set $V(Y)$ intersects at most $\delta(Y)$ of elementary cubes in each such row and there is at most $|Y|$ such rows that have a nonempty intersection with $V(Y)$. By the definition of $\chi_{q}^{\prime}(Y)$ and (A.11), we get

$$
\tilde{\beta}\left|f_{q}^{(n-1)}-f_{m}^{(n-1)}\right||V(Y)| \leqq(1+\alpha)|Y|
$$

for all $m \neq q$ As a consequence,

$$
a_{q}^{(n-1)}|V(Y)| \leqq(1+\alpha)|Y|,
$$

provided $\chi_{q}^{\prime}(Y) \neq 0$. Combined with $\left(\right.$ A.10) and the fact that $\chi_{q}^{\prime}(Y) \leqq 1$, this implies that

$$
\left|K_{q}^{\prime}(Y)\right| \leqq e^{-[\gamma-1-\alpha-O(\varepsilon)]|Y|},
$$

which yields the desired bound i) for $\delta(Y)=n$

Proof of ii) for $\delta(Y)=n$ Using (4 22), (2.7), (4.4), and (A.8), we get

$$
\begin{aligned}
\left|\frac{\partial}{\partial \mu_{i}}\left(\rho(Y) e^{\tilde{\beta} e_{q}|Y|}\right)\right| & \leqq\left(2 \tilde{\beta} C_{0}+1\right)|Y| e^{-\left(\tilde{\beta} e_{0}+\gamma\right)|Y|} e^{\tilde{\beta} e_{q}|Y|} \\
& \leqq\left(2 \tilde{\beta} C_{0}+1\right)|Y| e^{-(\gamma-O(\varepsilon))|Y|} e^{a_{q}^{(n-1)}|Y|}
\end{aligned}
$$


Using inductive assumption i) and ii) for contours contributing to $Z_{q}^{\prime}\left(\operatorname{Int}_{m} Y\right)$, we can apply the cluster expansion to get the bounds

$$
\left|\frac{\partial}{\partial \mu_{i}} \log Z_{q}^{\prime}\left(\operatorname{Int}_{m} Y\right)\right| \leqq\left[\tilde{\beta} C_{0}+\left(1+\tilde{\beta} C_{0}\right) O(\varepsilon)\right]\left|\operatorname{Int}_{m} Y\right|
$$

and

$$
\tilde{\beta}\left|\frac{\partial}{\partial \mu_{i}} f_{m}^{(n-1)}\right| \leqq\left[\tilde{\beta} C_{0}+\left(1+\tilde{\beta} C_{0}\right) O(\varepsilon)\right]
$$

Using further the inductive assumption vi) and vii), as well as the bounds (A.5) and (A.6), we get

$$
\begin{aligned}
\left|\frac{\partial}{\partial \mu_{i}} \prod_{m} \frac{Z_{m}\left(\operatorname{Int}_{m} Y\right)}{Z_{q}^{\prime}\left(\operatorname{Int}_{m} Y\right)}\right| & \leqq\left[\left(3 \tilde{\beta} C_{0}+1\right)+\left(1+\tilde{\beta} C_{0}\right) O(\varepsilon)\right]|\operatorname{Int} Y| e^{a_{q}^{(n-1)}|\operatorname{Int} Y|} e^{O(\varepsilon)|Y|} \\
& \leqq\left[\left(2 r \tilde{\beta} C_{0}+1\right)+\left(1+\tilde{\beta} C_{0}\right) O(\varepsilon)\right]|\operatorname{Int} Y| e^{a_{q}^{(n-1)}|\operatorname{Int} Y|} e^{O(\varepsilon)|Y|} .
\end{aligned}
$$

With the help of (A.16) and (5.11) we get

$$
\begin{aligned}
\left|\frac{\partial}{\partial \mu_{i}} \chi_{q}^{\prime}(Y)\right| & \leqq 2(r-1)\left[\tilde{\beta} C_{0}+\left(1+\tilde{\beta} C_{0}\right) O(\varepsilon)\right] \delta(Y) \\
& \leqq 2(r-1)\left[\tilde{\beta} C_{0}+\left(1+\tilde{\beta} C_{0}\right) O(\varepsilon)\right]|Y| .
\end{aligned}
$$

Combining now (A.14), (A.17), and (A.18) with (A.6), (A.9) and the observation that $|V(Y)|=|Y|+\mid$ Int $Y \mid$, we get

$$
\left|\frac{\partial}{\partial \mu_{i}} K_{q}^{\prime}(Y)\right| \leqq\left\{2 r \tilde{\beta} C_{0}+1+\left(1+\tilde{\beta} C_{0}\right) O(\varepsilon)\right\}|V(Y)| e^{a_{q}^{(n-1)}|V(Y)|} e^{-(\gamma-O(\varepsilon))|Y|} .
$$

Using now again the fact that $K_{q}^{\prime}(Y)$ (and its derivatives) vanishes unless (A.12) is fulfilled, we get the desired bound, provided $\varepsilon$ is sufficiently small.

Proof of iii) for $k=\delta(Y) \leqq n$ and $a_{q}^{(n)} \delta(Y) \leqq \bar{\alpha}$. We just have proved that i) is true for all contours $Y$ with $\delta(Y) \leqq n$. As a consequence, both $f_{m}^{(k-1)}$ and $f_{m}^{(n)}$ may be analyzed by a convergent cluster expansion. Using the definition of $f_{m}^{(n)}$ and the obvious fact that $|Y| \geqq \delta(Y)$ (again, $d \geqq 2$ ), one concludes that all contours $Y$ contributing to the cluster expansion of the difference $f_{m}^{(k-1)}-f_{m}^{(n)}$ obey the bound $|Y| \geqq k$. As a consequence,

$$
\tilde{\beta}\left|f_{m}^{(k-1)}-f_{m}^{(n)}\right| \leqq(K \varepsilon)^{k}
$$

and

$$
\tilde{\beta}\left|f_{m}^{(k-1)}-f_{m}^{(n)}\right| \delta(Y) \leqq(K \varepsilon)^{k} \delta(Y)=k(K \varepsilon)^{k} \leqq O(\varepsilon),
$$

where $K$ is a constant depending only on the dimension $d$ and the number of phases $r$. Combining (A.21) with the assumption $a_{q}^{(n)} \delta(Y) \leqq \bar{\alpha}$, we obtain the lower bound

$$
\alpha-\tilde{\beta}\left[f_{q}^{(k-1)}-f_{m}^{(k-1)}\right] \delta(Y) \geqq \alpha-a_{q}^{(n) \delta(Y)}-O(\varepsilon) \geqq \alpha-\bar{\alpha}-O(\varepsilon)=2-O(\varepsilon),
$$


with $\bar{\alpha}$ defined by (A 4) Combining this with (5.12) we get the equality $\chi_{q}^{\prime}(Y)=1$

Proof of iv) and $v$ ) The statement follows from the just proven fact that $\%_{q}^{\prime}(Y)=1$ for all contours $Y$ with $\delta(Y) a_{q}^{(n)} \leqq \bar{\alpha}$, the definiton (59) of $K_{q}^{\prime}(Y)$ and the relations (56) and (5.4) We proceed by a second induction on the diameters of $Y$ and $V$. For $\delta(Y)=0$ or $\delta(V)=0$ the statement is trivial Assume now that $K_{q}^{\prime}(Y)=K_{q}(Y)$ for all $Y$ with $\delta(Y) \leqq k<n$ and $\delta(Y) a_{q}^{(n)} \leqq \bar{\alpha}$. Comparing (54) and (5.6), we conclude that $Z_{q}^{\prime}(V)=Z_{q}(V)$ for all $V$ with $\delta(V) \leqq k<n$ and $\delta(V) a_{q}^{(n)} \leqq \bar{\alpha}$. Let $Y$ be a contour with $\delta(Y)=k+1$ and $\delta(Y) a_{q}^{(n)} \leqq \bar{\alpha}$ Then $Z_{q}^{\prime}\left(\operatorname{Int}_{m} Y\right)=Z_{q}\left(\operatorname{Int}_{m} Y\right)$ since $\delta\left(\operatorname{Int}_{m} Y\right) \leqq \delta(Y)-1=k$. Combined with the fact that $\chi_{q}^{\prime}(Y)=1$ by iii), we conclude that $K_{q}^{\prime}(Y)=K_{q}(Y)$. This completes the induction on $k$.

Proof of vi) for $\delta(V)=n$ We say that a contour $Y$ is small if $a_{q}^{(n)} \delta(Y) \leqq \bar{\alpha}$ while it is large if $a_{q}^{(n)} \delta(Y)>\bar{\alpha}$. We then rewrite the partition function $Z_{q}(V)$ given in the form (5 1$)$ by splitting the set of external contours into small and large contours and, for a fixed collection of large external contours $\left\{X_{1}, \quad, X_{k}\right\}_{\text {ext }}$, we resum over (mutually external) small $q$-contours in Ext $=V \backslash \bigcup_{l=1}^{k} V\left(X_{i}\right)$. As a result we get

$$
Z_{q}(V)=\sum_{\left\{X_{1}, X_{h}\right\}_{\mathrm{cxt}}} Z_{q}^{\text {small }}(\mathrm{Ext}) \prod_{l=1}^{k}\left[\rho\left(X_{i}\right) \prod_{m} Z_{m}\left(\operatorname{Int}_{m} X_{i}\right)\right]
$$

with the sum going over sets of mutually external large contours in $V$. The partition function $Z_{q}^{\text {small }}($ Ext $)$ is obtained from $Z_{q}($ Ext $)$ by dropping all large external $q$ contours

Due to the inductive assumption iv), $K_{q}(Y)=K_{q}^{\prime}(Y)$ if $Y$ is small Since $\left|K_{q}^{\prime}(Y)\right| \leqq \varepsilon^{|Y|}$ by i), $Z_{q}^{\text {small }}($ Ext $)$ can be evaluated by a convergent cluster expansion, and

$$
\mid Z_{q}^{\text {small }}(\text { Ext }) \mid \leqq e^{-\hat{\beta} \operatorname{Re} f_{q}^{\text {small }}|\operatorname{Ext}|} e^{O(\varepsilon)|\hat{c} \operatorname{Ext}|} .
$$

Here $f_{q}^{\text {small }}$ is the free energy of the contour model with activities

$$
K_{q}^{\text {small }}(Y)= \begin{cases}K_{q}^{\prime}(Y) & \text { if } \delta(Y) \leqq n \text { and } Y \text { is small } \\ 0 & \text { otherwise }\end{cases}
$$

On the other hand,

$$
\prod_{m}\left|Z_{m}\left(\operatorname{Int}_{m} X_{l}\right)\right| \leqq e^{-\tilde{\beta} f_{0}^{(n-1)}\left|\operatorname{Int} X_{l}\right|} e^{O(\varepsilon)\left|\hat{\operatorname{In} t} X_{l}\right|}
$$

by the induction assumption vi) Observing that the smallest contours contributing to the difference of $f_{m}^{(n)}$ and $f_{m}^{(n-1)}$ obey the bound $|Y| \geqq n$, while

$$
\left|V\left(X_{i}\right)\right| \leqq \delta\left(X_{l}\right)\left|X_{i}\right| \leqq n\left|X_{l}\right|,
$$

we may continue as in the proof of (A.21) to bound

$$
\tilde{\beta}\left|f_{0}^{(n-1)}-f_{0}^{(n)}\right|\left|\operatorname{Int} X_{l}\right| \leqq \tilde{\beta}\left|f_{0}^{(n-1)}-f_{0}^{(n)}\right|\left|V\left(X_{l}\right)\right| \leqq n(K \varepsilon)^{n}\left|X_{i}\right| \leqq O(\varepsilon)\left|X_{i}\right|
$$


Thus

$$
\prod_{m}\left|Z_{m}\left(\operatorname{Int} X_{i}\right)\right| \leqq e^{-\tilde{\beta} f_{0}^{(n)}\left|\operatorname{Int} X_{l}\right|} e^{O(\varepsilon)\left|X_{l}\right|}
$$

Combining (A.23) and (A.25) with the bounds

$$
\left|\rho\left(X_{i}\right)\right| \leqq e^{-\gamma\left|X_{i}\right|-\tilde{\beta} e_{0}\left|X_{i}\right|} \leqq e^{-(\gamma-O(\varepsilon))\left|X_{i}\right|} e^{-\tilde{\beta} f_{0}^{(n)}\left|X_{i}\right|}
$$

and

$$
|\partial \mathrm{Ext}| \leqq|\partial V|+\sum_{i=1}^{k}\left|\partial V\left(X_{i}\right)\right| \leqq|\partial V|+2(d+1) \sum_{i=1}^{k}\left|X_{i}\right|
$$

we conclude that

$$
\left|Z_{q}(V)\right| \leqq e^{O(\varepsilon)|\partial V|} e^{-\tilde{\beta} f_{0}^{(n)}|V|} \sum_{\left\{X_{1}, X_{k}\right\}_{\mathrm{ext}}} e^{-\tilde{\beta}\left[\operatorname{Re} f_{q}^{\text {small }}-\operatorname{Re} f_{q}^{(n)}\right]|\operatorname{Ext}|} \prod_{i=1}^{k} e^{-(\gamma-O(\varepsilon))\left|X_{i}\right|}
$$

Next, we bound the difference $f_{q}^{\text {small }}-f_{q}^{(n)}$. First, for all large contours $X$, we have

$$
|X| \geqq \delta(X) \geqq l_{0}:=\frac{\bar{\alpha}}{a_{q}^{(n)}} .
$$

Next, we observe that

$$
\tilde{\beta}\left|f_{q}^{(n)}-f_{q}^{\text {small }}\right| \leqq(K \varepsilon)^{l_{0}} \leqq \frac{1}{-l_{0} \log (K \varepsilon)},
$$

where $K$ is a constant depending only on $d$ and $N$. Recalling the condition $\bar{\alpha} \geqq 1$, we get

$$
\tilde{\beta}\left|f_{q}^{(n)}-f_{q}^{\text {small }}\right| \leqq \frac{a_{q}^{(n)}}{-\log (K \varepsilon)} \leqq \frac{1}{2} a_{q}^{(n)}
$$

provided $\varepsilon$ is chosen small enough. Combining (A.28) with (A.31), we finally obtain

$$
\left|Z_{q}(V)\right| \leqq e^{O(\varepsilon)|\partial V|} e^{-\tilde{\beta} f_{0}^{(n)}|V|} \sum_{\left\{X_{1}, X_{k}\right\}_{\mathrm{ext}}} e^{-\frac{a_{q}^{(n)}}{2}|\operatorname{Ext}|} \prod_{i=1}^{k} e^{-\tilde{\gamma}\left|X_{i}\right|}
$$

with

$$
\tilde{\gamma}=\gamma-1 \text {. }
$$

At this point we need the following Lemma A.2, which is a variant of a lemma first proven in [Zah84] (see also [BI89] and [BK94] for the proof of this lemma exactly in the following formulation).

Lemma A.2. Consider an arbitrary contour activity $\tilde{K}_{q}(Y) \geqq 0$, and let $\tilde{Z}_{q}$ be the partition function

$$
\tilde{Z}_{q}(V)=\sum_{\left\{Y_{1},, Y_{n}\right\}} \prod_{i=1}^{n}\left(\tilde{K}_{q}\left(Y_{i}\right) e^{\left|Y_{1}\right|}\right)
$$

Let $\tilde{s}_{q}$ be the corresponding free energy, and assume that $\tilde{K}_{q}(Y) \leqq \tilde{\varepsilon}^{|Y|}$, where $\tilde{\varepsilon}$ is small (depending on $r$ and $d$ ). Then for any $\tilde{a} \geqq-\tilde{s}_{q}$ the following bound 
is true

$$
\sum_{\left\{Y_{1}, Y_{k}\right\}_{\mathrm{cxt}}} e^{\tilde{a}|\operatorname{Ext}|} \prod_{l} \tilde{K}_{q}\left(Y_{i}\right) \leqq e^{O(\tilde{\varepsilon})|\hat{c} V|},
$$

with the sum running over all sets of mutually external q-contours in $V$

In order to apply the lemma, we define $\tilde{K}_{q}(Y)=e^{-\tilde{\tau}|Y|}$ if $Y$ is a large $q$-contour, and $\tilde{K}_{q}(Y)=0$ otherwise. With this choice,

$$
0 \leqq-\tilde{s}_{q} \leqq(K \varepsilon)^{l_{0}} \leqq \frac{1}{-l_{0} \log (K \varepsilon)}
$$

As a consequence,

$$
-\tilde{s}_{q} \leqq \tilde{a} \cdot=\frac{a_{q}^{(n)}}{2},
$$

provided $\varepsilon$ is small enough. Applying Lemma A 2 to the right-hand side of (A 32), and observing that $\tilde{\varepsilon} .=e^{-\tau} \leqq \varepsilon$, we finally obtain the desired inequality

$$
\left|Z_{q}(V)\right| \leqq e^{O(\varepsilon)|\hat{C} V|} e^{-\tilde{\beta} f_{0}^{(n)}|V|}
$$

Proof of vii) for $\delta(V)=n$ Beginning from the formula (A 22) above, we first notice that

$$
\mid \frac{\partial}{\partial \mu_{l}} \log Z_{q}^{\text {small }}(\text { Ext } Y)\left|\leqq\left(\tilde{\beta} C_{0}+\left(1+\tilde{\beta} C_{0}\right) O(\varepsilon)\right)\right| \text { Ext } Y \mid
$$

(cf (A 15)) since we can use ii) for small $Y$ Using further the bound (A.23) we can conclude that

$$
\mid \frac{\partial}{\partial \mu_{l}} Z_{q}^{\text {small }}(\text { Ext })\left|\leqq\left(\tilde{\beta} C_{0}+\left(1+\tilde{\beta} C_{0}\right) O(\varepsilon)\right)\right| \operatorname{Ext} Y \mid e^{-\tilde{\beta} \operatorname{Re} f_{q}^{\text {small }}|\operatorname{Ext}|} e^{O(\varepsilon)|\hat{E x t}|}
$$

Combining this with the bounds (4 22) as well as (A 23) for $Z_{q}^{\text {small }}$ (Ext) and the inductive assumptions (vii) and (vi) for $Z_{m}\left(\operatorname{Int}_{m} X_{l}\right)$, we get

$$
\begin{aligned}
& \left|\frac{\partial}{\partial \mu_{i}} Z_{q}(V)\right| \leqq e^{O(\varepsilon)|\hat{c} V|} e^{-\tilde{\beta} f_{0}^{(n)}|V|} \sum_{\left\{X_{1},, X_{k}\right\}_{\mathrm{cxt}}}\left|\left(\tilde{\beta} C_{0}+\left(1+\tilde{\beta} C_{0}\right) O(\varepsilon)\right)\right| \operatorname{Ext} Y \mid \\
& \quad+\left(2 \tilde{\beta} C_{0}+1\right)|\operatorname{Int} Y|+\left(\tilde{\beta} C_{0}+1\right)|Y| \mid e^{-\tilde{\beta}\left[\operatorname{Re} f_{q}^{\text {small }}-\operatorname{Re} f_{q}^{(n)}\right]|\operatorname{Ext}|} \prod_{i=1}^{k} e^{-(\gamma-O(\varepsilon))\left|X_{t}\right|}
\end{aligned}
$$

Proceeding now as in the proof of vi) from (A.28), we finally get vii) This concludes the inductive proof of Lemma A 1.

For $V=\mathbb{T}_{A}$, the bound from Lemma A 1 vi) can be actually strengthened.

Lemma A.3. Under the assumptions of Lemma A 1 we have

$$
\left|Z_{q}\left(\mathbb{T}_{1}\right)\right| \leqq e^{-\tilde{\beta} f_{0}\left|\mathbb{T}_{1}\right|} e^{O(\varepsilon)\left|\hat{\tilde{T}} \mathbb{T}_{1}\right|} \max \left\{e^{-\frac{u_{q}}{4}\left|\mathbb{T}_{1}\right|}, e^{-K(d) ;\left|\hat{\mathbb{T}} \mathbb{T}_{1}\right|}\right\}
$$

with a constant $K(d)$ that depends only on the dimension $d$ 
Proof. Extracting from (A.32) the factor $\max _{\left\{X_{1}, X_{k}\right\}_{\text {ext }}} e^{-\frac{a_{q}^{(n)}}{4} \mid \text { Ext } \mid} \prod_{i=1}^{k} e^{-\frac{1}{2} \tilde{\gamma}\left|X_{i}\right|}$, we still get the same bound. This factor can be, the limit $n \rightarrow \infty$, clearly bounded by $\max _{U \subset V}\left(e^{-\frac{a_{q}}{4}|V \backslash U|} e^{-K(d) \gamma|\partial U|}\right)$. As a consequence,

$$
\left|Z_{q}(V)\right| \leqq e^{-\tilde{\beta} f_{0}|V|} e^{O(\varepsilon)|\partial V|} \max _{U \subset V}\left(e^{-\frac{a_{q}}{4}|V \backslash U|} e^{-K(d) \gamma|\partial U|}\right),
$$

which is still true for arbitrary $V \subset \mathbb{T}$. We now decompose $U$ and $V$ into time slices, $U=\bigcup_{t} U_{t}$ and $V=\sum_{t} V_{t}$, and observe that $|V \backslash U|=\sum_{t}\left(\left|V_{t}\right|-\left|U_{t}\right|\right)$, while $|\partial U| \geqq \sum_{t}\left|\partial \pi\left(U_{t}\right)\right|$, where $\pi(\cdot)$ denotes the projection onto $\mathbb{R}^{d}$. Using the isoperimetric inequality on $\mathbb{R}^{d}$, we now bound

$$
\begin{aligned}
\max _{U \subset V}\left(e^{-\frac{a_{q}}{4}|V \backslash U|} e^{-2 d K(d) \gamma|U|^{\frac{d-1}{d}}}\right) & \leqq \prod_{t=1}^{M} \max _{U_{t} \subset V_{t}}\left(e^{-\frac{a_{q}}{4}\left(\left|V_{t}\right|-\left|U_{t}\right|\right)} e^{-2 d K(d) \gamma\left|U_{t}\right|^{\frac{d-1}{d}}}\right) \\
& \leqq \prod_{t=1}^{M} \max \left\{e^{-\frac{a_{q}}{4}\left|V_{t}\right|}, e^{-2 d K(d) \gamma\left|V_{t}\right|^{\frac{d-1}{d}}}\right\}
\end{aligned}
$$

Restricting ourselves to $V=\mathbb{T}_{\Lambda}$, we observe that $\left|V_{t}\right|=|V| / M$ is independent of $t$ in this case. As consequence,

$$
\begin{aligned}
\max _{U \subset V}\left(e^{-\frac{a_{q}}{4}|V \backslash U|} e^{-2 d K(d) \gamma|U|^{\frac{d-1}{d}}}\right) & \leqq\left(\max \left\{e^{-\frac{a_{q}}{4}|V| / M}, e^{-2 d K(d) \gamma(|V| / M)^{\frac{d-1}{d}}}\right\}\right)^{M} \\
& =\max \left\{e^{-\frac{a_{q}}{4}|V|}, e^{-K(d) \gamma|\partial V|}\right\}
\end{aligned}
$$

where we used that $|\partial V|=2 d M(|V| / M)^{\frac{d-1}{d}}$ in the last step.

\section{References}

[Bor88] Borgs, C: Confinement, Deconfinement and Freezing in Lattice Yang-Mills theories with Continuous Time Commun Math Phys. 116, 309-342 (1988)

[BI89] Borgs, C., Imbrie, J : A Unified Approach to Phase Diagrams in Field Theory and Statistical Mechanics Commun. Math Phys 123, 305-328 (1989)

[BK90] Borgs, C, Kotecký, R : A Rigorous Theory of Finite Size Scaling at First Order Phase Transitions J Stat Phys 61, 79-119 (1990)

[BK94] Borgs, C, Kotecký, R : Surface induced finite size effects for first order phase transitions J Stat Phys 79, 43-115 (1995)

[BK95] Borgs, C., Kotecký, R.: Low Temperature Phase Diagrams of Fermionic Lattice Systems (1995), in preparation

[DFF95] Datta, N., Fernandez, R, Fröhlich, J : Stat Phys, in print (1995)

[Dob65] Dobrushin, R L: Existence of a phase transition in the two-dimensional and threedimensional Ising models Soviet Phys Doklady 10, 111-113 (1965)

[Dob94] Dobrushin, R L.: Estimates of Semiinvariants for the Ising Model at Low Temperatures (1994) Preprint ESI 125, available from FTP ESI AC AT

[GKK88] Gawędzki, K, Kotecký, R, Kupiainen, A : Coarse-Graining Approach to First-Order Phase Transitions J Stat Phys 47, 701-724 (1988)

[Gin69] Ginibre, J : Existence of phase transitions for quantum lattice systems Commun. Math. Phys. 14, 205 (1969)

[Gri64] Griffiths, R.B : Peierls proof of spontaneous magnetization in a two-dimensional Ising ferromagnet Physical Review A 136, 437-439 (1964)

[Ken85] Kennedy, T : Long Range Order in the Anisotropic Quantum Ferromagnetic Heisenberg Model Commun. Math Phys 100, 447-462 (1985) 
[KP84] Kotecký, R, Preiss, D : An Inductive Approach to the Pirogov-Sinai Theory Suppl ai Rendiconti del Circolo Matem di Palermo, Ser II 3, 161-164 (1984)

[Pei36] Peierls, R: On the Ising model of ferromagnetism Proc Camb Philo Soc 32, 477-481 (1936)

[Pir78] Pirogov, S A : Phase diagrams of quantum lattice systems Soviet Math Dokl 19, 1096$1099(1978)$

[PS75] Pirogov, S A, Sinai, Ya G: Phase diagrams of classical lattice systems Theor and Math Phys 25, 1185-1192 (1975); 26, 39-49 (1976)

[Sei82] Seiler, E: Gauge Theories as a Problem of Constructive Quantum Field Theory and Statistical Mechanics Lecture Notes in Physics 159 Berlin-Heidelberg-etc SpringerVerlag (1982)

[Sin82] Sinai, Ya G: Theory of phase transitions: Rigorous results Oxford-New York-ctc Pergamon Press (1982)

[SS76] Seiler, E, Simon B : Nelson's symmetry and all that in Yukawa and $\left(\phi^{4}\right)_{3}$ theories Ann Phys 97, 470 (1976)

[Zah84] Zahradník, M: An Alternate Version of Pirogov-Sinai Theory Commun Math Phys 93, 559-581 (1984)

Communicated by Ya G Sinai 